\title{
Adaptive Backstepping Based MTPA Sensorless Control of PM-Assisted SynRM with Fully Uncertain Parameters
}

\author{
Yang Yu (D), ${ }^{1}$ Da Chang, ${ }^{1}$ Xiaoming Zheng, ${ }^{2}$ Zengqiang Mi, ${ }^{2}$ Xiaolong Li, $^{3}$ and Chenjun Sun ${ }^{3}$ \\ ${ }^{1}$ Hebei Key Laboratory of Distributed Energy Storage and Micro-Grid, North China Electric Power University, Baoding, \\ Hebei 071003, China \\ ${ }^{2}$ State Key Laboratory of Alternate Electrical Power System with Renewable Energy Sources, North China Electric Power University, \\ Baoding 071003, China \\ ${ }^{3}$ Department of Technology and Information, State Grid Hebei Electric Power Company, Shijiazhuang, Hebei 050022, China
}

Correspondence should be addressed to Yang Yu; ncepu_yy@163.com

Received 5 September 2017; Revised 3 December 2017; Accepted 25 December 2017; Published 29 January 2018

Academic Editor: Ton D. Do

Copyright (C) 2018 Yang Yu et al. This is an open access article distributed under the Creative Commons Attribution License, which permits unrestricted use, distribution, and reproduction in any medium, provided the original work is properly cited.

\begin{abstract}
A nonlinear and robust adaptive backstepping based maximum torque per ampere speed sensorless control scheme with fully uncertain parameters is proposed for a permanent magnet-assisted synchronous reluctance motor. In the design of the controller, the relation to $d$-q-axis currents constrained by maximum torque per ampere control is firstly derived. Then, a fully adaptive backstepping control method is employed to design control scenario and the stability of the proposed control scenario is proven through a proper Lyapunov function candidate. The derived controller guarantees tracking the reference signals of change asymptotically and has good robustness against the uncertainties of motor parameters and the perturbation of load torque. Moreover, in allusion to the strong nonlinearity of permanent magnet-assisted synchronous reluctance motor, an active flux based improved reduced-order Luenberger speed observer is presented to estimate the speed. Digital simulations testify the feasibility and applicability of the presented control scheme.
\end{abstract}

\section{Introduction}

Drive system is vital for various industrial applications. One of the important trends for the drive field is that direct current (DC) motor drive systems are being replaced by alternate current (AC) motor drive system due to low cost, convenient control, and superior performance of AC motor drive systems. A permanent magnet-assisted synchronous reluctance motor (PMa-SynRM) based drive system is a unique state-ofthe-art technology, where permanent magnets are equipped in magnetic barrier of rotor. Permanent magnet synchronous motor (PMSM) incorporated with synchronous reluctance motor makes PMa-SynRM appeal to the public in high efficiency, good utilization of the inverter, and wide range of flux-weak regulation. The uniqueness in structure of PMaSynRM attracts the investigator's attention of optimizing the design of motor body and putting forward more diverse and fascinating structures $[1,2]$.
As for its control, it is a bottleneck problem researched insufficiently. The synchronous change of speed and frequency for PMa-SynRM makes speed be regulated by adjusting voltage or frequency, which leads to three commonly used approaches to control: voltage-to-frequency control (VFC), direct torque control (DTC), and vector control (VC). VFC is a scalar and open-loop control, and its voltage regulated by space vector modulation always follows the change of given value. Low cost and ease of use are the prominent superiorities of VFC [3]. However, the weaknesses of slow response, poor performance of control, and insufficient utilization of torque hinder its successful development. Takahashi and Noguchi [4] and Depenbrock [5] studied DTC about 30 years ago, which was applied to control asynchronous motor first and was gradually introduced in PMSM until it had been developed and matured [6]. In terms of space vector and stator flux orientation, a hysteresis comparator is designed to control flux and torque. Through comparison of feedback 
value and given value in flux and torque, respectively, the states of converter switches are produced and torque output with high performance and rapid response is gained ultimately. Definite physical meaning of control signals and quick torque response are the remarkable assets for DTC. But torque ripple, current impulse, and massive online computations restrict its application [7]. VC is originated from the principle of flux orientation and enables controlling flux and torque to be decoupled like DC motor [8]. Therefore, it is famous for flexibility, high accuracy, and stable performance in low speed. For PMa-SynRM, maximum torque per ampere (MTPA) vector control has great advantages of large torque in low speed, small capacity in inverter unit, and facility to utilize reluctance torque compared to $i_{d}=0$ vector control $[9,10]$. The essence of MTPA control is to allocate $d$-q-axis currents appropriately. The relationship of constrained $d-q$ axis currents in MTPA principle is multiple, high-order, and nonlinear. The $d$-q-axis reference currents predetermined through offline method are a good solution. One of the representative offline methods is look-up table method [11], which is easy to execute but adds burden to store and requires extra offline effort [12]. Additionally, conventional MTPA control relies heavily on precise modeling parameters. Variation of parameters directly worsens its control performance. Especially for PMa-SynRM, previous investigations clearly demonstrate that its resistance, inductance, and flux linkage are directly related to operating temperature [13]. Hence, research on MTPA control with uncertain parameters is a hot and thorny issue. Niazi and Toliyat [14] estimated $d$-q-axis inductances and flux linkage of PMa-SynRM under multiple reference frame and [15] proposed a robust MTPA control scheme of PMa-SynRM for variation of $d$-q-axis inductances. The actuality is that only partial system uncertainties are considered, which desires the other system parameters to be known.

Backstepping control (BC) is a new style recursive technology for uncertain nonlinear plant $[16,17]$. With the aid of virtual control variables and plenty of recursive steps, the ultimate controller can be completed systematically and original high-order plant is reduced to a lower dimension. Furthermore, integration of backstepping control with adaptive control and uncertain plant parameters can be estimated through selecting a suitable Lyapunov function. A robust nonlinear controller based on BC and MTPA has been proposed for speed control of interior PMSM [18, 19]. Nevertheless, to the best of our knowledge, previous researches on employment of BC into PMa-SynRM have not been found. A majority of control schemes utilize conventional PID control [20]. Moreover, three intrinsic deficiencies in traditional BC should be mentioned as follows:

(A1) Linear treatment of uncertain plant parameters results in solving highly complicated regression matrices in the design of control; utilization of various linearization theories may ignore some beneficial nonlinearities of the plant.

(A2) Partial parameters uncertainties considered cause to the designed control scenario vulnerable to variation of extra values.
(A3) Determination of control parameters in the last time derivative of ultimate Lyapunov function requires the positive definite terms which only include partial model information. Once the derivative is induced to be positive definite by these positive terms, the asymptotic stability of the controller will be damaged and unfortunately it is nearly impossible to discover.

The innovation of the study is firstly to propose a new nonlinear and fully adaptive BC approach with no problems of overparameterization and singularity for an uncertain PMa-SynRM and hence (A1) is excluded. Previous researches such as that in [21] cannot resolve full parameter uncertainties, and the problems of overparameterization and singularity cannot be complete to be coped with. Furthermore, the paper enables overcoming the shortcomings of conventional $\mathrm{BC}$, and totally seven parameter uncertainties of six motor parameters and one load parameter are taken into account, which construct a full adaptive structure and eliminate (A2) and (A3). Merely the number of pole pairs is considered to be known, due to the fact that it does not change in operation and can be acquired from nameplate. The other novelty in the context is that adaptive $\mathrm{BC}$ is introduced into MTPA control through implicit and symbolic computation methodology. Additionally, an active flux based improved reduced-order Luenberger speed observer is developed to estimate the speed of PMa-SynRM, resisting its strong and high nonlinearity of dynamic model. The remainder of the paper is arranged as follows: the dynamics of PMa-SynRM with fully uncertain parameters are introduced in Section 2. The relation constrained in $d$-q-axis currents under MTPA control is explicitly derived in Section 3. Section 4 discloses the design scenario of the proposed controller with relation to Lyapunov stability analysis. An improved Luenberger speed observer on the basis of active flux is developed in Section 5 to estimate the speed. The effectiveness and correctness of the proposed algorithm are validated in Section 6 and the simulation results are discussed. The final section sums up the paper.

\section{Dynamics of PMa-SynRM with Fully Uncertain Parameters}

The model of a typical PMa-SynRM is expressed in rotating $d$-q-axis coordinate system as follows [22]:

Stator Voltage Equations

$$
\begin{aligned}
& u_{d}=R_{s} i_{d}+\frac{d \psi_{d}}{d t}-n_{p} \omega_{r} \psi_{q}, \\
& u_{q}=R_{s} i_{q}+\frac{d \psi_{q}}{d t}+n_{p} \omega_{r} \psi_{d} .
\end{aligned}
$$

Flux Linkage Equations

$$
\begin{aligned}
& \psi_{d}=L_{d} i_{d}, \\
& \psi_{q}=L_{q} i_{q}-\psi_{f} .
\end{aligned}
$$


Electromagnetic Torque

$$
T_{e}=\frac{3}{2} \frac{n_{p}}{2}\left[\psi_{f} i_{d}+\left(L_{d}-L_{q}\right) i_{d} i_{q}\right]
$$

Thus, the dynamic model of a PMa-SynRM can be described as

$$
\begin{aligned}
\frac{d i_{d}}{d t} & =-\frac{R_{s}}{L_{d}} i_{d}+\frac{n_{p} L_{q}}{L_{d}} \omega_{r} i_{q}-\frac{n_{p} \psi_{f}}{L_{d}} \omega_{r}+\frac{1}{L_{d}} u_{d}, \\
\frac{d i_{q}}{d t} & =-\frac{R_{s}}{L_{q}} i_{q}-\frac{n_{p} L_{d}}{L_{q}} \omega_{r} i_{d}+\frac{1}{L_{q}} u_{q}, \\
\frac{d \omega_{r}}{d t} & =\frac{1}{J_{m}}\left(T_{e}-B_{m} \omega_{r}-T_{L}\right),
\end{aligned}
$$

where $i_{d}$ and $i_{q}$ are the $d$-q-axis currents, $u_{d}$ and $u_{q}$ are the $d$ - $q$-axis voltages, $\psi_{d}$ and $\psi_{q}$ are the $d$ - $q$-axis flux linkages, $L_{d}$ and $L_{q}$ are the $d$-q-axis inductors, $R_{s}$ is the stator resistance, $n_{p}$ is the number of pole pairs, $\omega_{r}$ is the mechanical speed of rotor, $\psi_{f}$ is the flux linkage of permanent magnet, $J_{m}$ is the moment of inertia of rotor, $B_{m}$ is the viscous damping coefficient, and $T_{L}$ is the load torque denoting external torque disturbance.

Due to the existence of product terms between electrical stator currents and mechanical rotor speed in (5) and (6), PMa-SynRM as an electromechanical coupling system belongs to a highly nonlinear plant. With regard to the various electrical parameters $R_{s}, L_{d}, L_{q}$, and $\psi_{f}$, it should be noted that they could be directly measured or calculated, but their values may vary with operating and experimental conditions such as temperature, humidity, and flux saturation. The remaining mechanical parameters $J_{m}$ and $B_{m}$ are even more difficult, which are improbable to measure or calculate. The final load torque $T_{L}$ is frequently uncertain. It follows that PMa-SynRM suffers from inevitable parameter uncertainties and immeasurable disturbances. For this purpose, nonlinear adaptive control should be introduced to eliminate uncertainties and reject disturbances.

\section{Parameter Constraint Relation in MTPA Control}

The key of MTPA is to pursue electromagnetic torque as large as possible through reasonable distribution of $d$-q-axis stator currents. This problem can be transformed into an extreme problem in the following:

$$
\begin{aligned}
\min : & i_{s}=\sqrt{i_{d}^{2}+i_{q}^{2}}, \\
\text { condition: } & T_{e}=\frac{3}{2} \frac{n_{p}}{2}\left[\psi_{f} i_{d}+\left(L_{d}-L_{q}\right) i_{d} i_{q}\right],
\end{aligned}
$$

where $i_{s}$ is the stator current.

Through introduction of a Lagrangian multiplier $\xi$, the extreme problem given in (8) is converted into an auxiliary function solution problem in the following:

$$
F=\sqrt{i_{d}^{2}+i_{q}^{2}}+\xi\left\{T_{e}-\frac{3}{2} \frac{n_{p}}{2}\left[\psi_{f} i_{d}+\left(L_{d}-L_{q}\right) i_{d} i_{q}\right]\right\} .
$$

Using partial derivatives of $i_{d}, i_{q}$, and $\xi$ in (9), respectively, we can obtain

$$
\begin{aligned}
& \frac{\partial F}{\partial i_{d}}=\frac{i_{d}}{\sqrt{i_{d}^{2}+i_{q}^{2}}}-\frac{3}{4} \xi n_{p}\left[\psi_{f}+\left(L_{d}-L_{q}\right) i_{q}\right], \\
& \frac{\partial F}{\partial i_{q}}=\frac{i_{q}}{\sqrt{i_{d}^{2}+i_{q}^{2}}}-\frac{3}{4} \xi n_{p}\left(L_{d}-L_{q}\right) i_{d} \\
& \frac{\partial F}{\partial \xi}=T_{e}-\frac{3}{2} \frac{n_{p}}{2}\left[\psi_{f} i_{d}+\left(L_{d}-L_{q}\right) i_{d} i_{q}\right] .
\end{aligned}
$$

Let (10) be equal to zero; then the relation constrained in $d$-q-axis currents in MTPA control can be derived as

$$
i_{q}=-\frac{\psi_{f}}{2\left(L_{d}-L_{q}\right)}+\sqrt{i_{d}^{2}+\left(\frac{\psi_{f}}{2\left(L_{d}-L_{q}\right)}\right)^{2}} .
$$

Equation (11) indicates that if $i_{d}$ is acquired, $i_{\text {qref }}$ will be determined.

\section{Nonlinear Adaptive Backstepping Controller}

The control target of the study is to guarantee all the signals to be bounded and ensures the speed and currents to track their respective references precisely and rapidly in spite of full parameter uncertainties in PMa-SynRM and load disturbance. For achievement of the goal, the designed controller enables tracking the variations of parameters uncertainties. Hence, online parameter estimation laws for fully uncertain parameters with no problems of overparameterization and singularity should be conducted and vary with actual parameters adaptively.

4.1. Designed Nonlinear and Fully Adaptive Backstepping Controller. The tracking errors $e_{\omega}, e_{d}$, and $e_{q}$ of $\omega_{r}, i_{d}$, and $i_{q}$ are defined as follows:

$$
\begin{aligned}
& e_{\omega}=\omega_{\text {ref }}-\omega_{r}, \\
& e_{d}=i_{d \mathrm{ref}}-i_{d}, \\
& e_{q}=i_{q \mathrm{ref}}-i_{q},
\end{aligned}
$$

where $\omega_{\text {ref }}, i_{d \text { ref }}$, and $i_{q \text { ref }}$ are the references of $\omega_{r}, i_{d}$, and $i_{q}$, respectively.

Step 1. By derivative of $e_{\omega}$ in (12) and integration of (13) and (14), the dynamics of speed tracking errors can be given by

$$
\begin{aligned}
\dot{e}_{\omega} & =\dot{\omega}_{\mathrm{ref}}-\dot{\omega}_{r}=\dot{\omega}_{\mathrm{ref}}-\frac{T_{e}}{J_{m}}+\frac{B_{m} \omega_{r}}{J_{m}}+\frac{T_{L}}{J_{m}}=\dot{\omega}_{\mathrm{ref}} \\
& -\frac{3 n_{p}\left[\psi_{f} i_{d}+\left(L_{d}-L_{q}\right) i_{d} i_{q}\right]}{4 J_{m}}+\frac{B_{m} \omega_{r}}{J_{m}}+\frac{T_{L}}{J_{m}} \\
& =\dot{\omega}_{\mathrm{ref}}+\frac{B_{m} \omega_{r}}{J_{m}}+\frac{T_{L}}{J_{m}}-\frac{3 n_{p}}{4 J_{m}}\left[\psi_{f} i_{d \mathrm{ref}}+\left(L_{d}-L_{q}\right)\right.
\end{aligned}
$$




$$
\begin{aligned}
& \left.\cdot i_{d \mathrm{ref}} i_{q \mathrm{ref}}\right]+\frac{3 n_{p}}{4 J_{m}}\left[\psi_{f}+\left(L_{d}-L_{q}\right) i_{q \mathrm{ref}}\right] e_{d} \\
& +\frac{3 n_{p}}{4 J_{m}}\left(L_{d}-L_{q}\right) i_{d \mathrm{ref}} e_{q}-\frac{3 n_{p}}{4 J_{m}}\left(L_{d}-L_{q}\right) e_{d} e_{q} \\
& =\frac{\left[\psi_{f}+\left(L_{d}-L_{q}\right) i_{q \mathrm{ref}}\right]}{J_{m}}\left(\frac{J_{m}}{\left[\psi_{f}+\left(L_{d}-L_{q}\right) i_{q \mathrm{ref}}\right]}\right. \\
& \cdot \dot{\omega}_{\mathrm{ref}}+\frac{B_{m}}{\left[\psi_{f}+\left(L_{d}-L_{q}\right) i_{q \mathrm{ref}}\right]} \omega_{r} \\
& +\frac{T_{L}}{\left[\psi_{f}+\left(L_{d}-L_{q}\right) i_{q \mathrm{ref}}\right]}-\frac{3 n_{p}}{4} i_{d \mathrm{ref}}+\frac{3 n_{p}}{4} e_{d} \\
& +\frac{3 n_{p}\left(L_{d}-L_{q}\right)}{4\left[\psi_{f}+\left(L_{d}-L_{q}\right) i_{q \mathrm{ref}}\right]} i_{d \mathrm{ref}} e_{q} \\
& \left.+\frac{3 n_{p}\left(L_{d}-L_{q}\right)}{4\left[\psi_{f}+\left(L_{d}-L_{q}\right) i_{q \mathrm{ref}}\right]} e_{d} e_{q}\right) .
\end{aligned}
$$

Supposing that $B_{m} /\left[\psi_{f}+\left(L_{d}-L_{q}\right) i_{q \text { ref }}\right]=\theta_{1}, T_{L} /\left[\psi_{f}+\left(L_{d}-\right.\right.$ $\left.\left.L_{q}\right) i_{\text {qref }}\right]=\theta_{2},\left(L_{d}-L_{q}\right) /\left[\psi_{f}+\left(L_{d}-L_{q}\right) i_{\text {qref }}\right]=\theta_{3}$, and $J_{m} /\left[\psi_{f}+\right.$ $\left.\left(L_{d}-L_{q}\right) i_{q r e f}\right]=\theta_{4}$, (15) can be rearranged as

$$
\begin{aligned}
& \dot{e}_{\omega}=\frac{1}{\theta_{4}}\left(\left(\hat{\theta}_{4}-\tilde{\theta}_{4}\right) \dot{\omega}_{\mathrm{ref}}+\left(\hat{\theta}_{1}-\tilde{\theta}_{1}\right) \omega_{r}+\left(\hat{\theta}_{2}-\tilde{\theta}_{2}\right)\right. \\
& -\frac{3 n_{p}}{4} i_{d \mathrm{ref}}+\frac{3 n_{p}}{4} e_{d}+\frac{3 n_{p}}{4}\left(\hat{\theta}_{3}-\widetilde{\theta}_{3}\right) i_{d \mathrm{ref}} e_{q} \\
& \left.-\frac{3 n_{p}}{4}\left(\widehat{\theta}_{3}-\widetilde{\theta}_{3}\right) e_{d} e_{q}\right)=\frac{1}{\theta_{4}}\left(\left(\widehat{\theta}_{4} \dot{\omega}_{\text {ref }}-\widetilde{\theta}_{4} \dot{\omega}_{\text {ref }}\right)\right. \\
& +\left(\widehat{\theta}_{1} \omega_{r}-\widetilde{\theta}_{1} \omega_{r}\right)+\left(\widehat{\theta}_{2}-\widetilde{\theta}_{2}\right)-\frac{3 n_{p}}{4} i_{d \mathrm{ref}}+\frac{3 n_{p}}{4} e_{d} \\
& \left.+\frac{3 n_{p}}{4}\left(\widehat{\theta}_{3} i_{d \mathrm{ref}} e_{q}-\tilde{\theta}_{3} i_{d \mathrm{ref}} e_{q}\right)-\frac{3 n_{p}}{4}\left(\widehat{\theta}_{3}-\tilde{\theta}_{3}\right) e_{d} e_{q}\right) \\
& =\frac{1}{\theta_{4}}\left(\left(\widehat{\theta}_{4} \dot{\omega}_{\text {ref }}+\widehat{\theta}_{1} \omega_{r}+\widehat{\theta}_{2}-\frac{3 n_{p}}{4} i_{d \mathrm{ref}}\right)-\left(\widetilde{\theta}_{4} \dot{\omega}_{\mathrm{ref}}\right.\right. \\
& +\widetilde{\theta}_{1} \omega_{r}+\widetilde{\theta}_{2}-\frac{3 n_{p}}{4} e_{d}-\frac{3 n_{p}}{4} \widehat{\theta}_{3} i_{d \mathrm{ref}} e_{q} \\
& \left.\left.+\frac{3 n_{p}}{4} \widetilde{\theta}_{3} i_{d \mathrm{ref}} e_{q}+\frac{3 n_{p}}{4}\left(\widehat{\theta}_{3}-\widetilde{\theta}_{3}\right) e_{d} e_{q}\right)\right) \text {, }
\end{aligned}
$$

where $\widehat{\theta}_{1}=\widetilde{\theta}_{1}+\theta_{1}, \widehat{\theta}_{2}=\widetilde{\theta}_{2}+\theta_{2}, \widehat{\theta}_{3}=\widetilde{\theta}_{3}+\theta_{3}$, and $\widehat{\theta}_{4}=\widetilde{\theta}_{4}+\theta_{4}$, $\widehat{\theta}_{1}, \widehat{\theta}_{2}, \widehat{\theta}_{3}$, and $\widehat{\theta}_{4}$ are the estimations of $\theta_{1}, \theta_{2}, \theta_{3}$, and $\theta_{4}$, and $\widetilde{\theta}_{1}, \widetilde{\theta}_{2}, \widetilde{\theta}_{3}$, and $\widetilde{\theta}_{4}$ are the estimation errors of $\theta_{1}, \theta_{2}, \theta_{3}$, and $\theta_{4}$.
Through some mathematical operations, (16) is arranged and consisted of two parts. In terms of (16), selection of $i_{d \text { ref }}$ is depicted in the following:

$$
i_{d \mathrm{ref}}=\frac{4}{3 n_{p}}\left(\widehat{\theta}_{4} \dot{\omega}_{\mathrm{ref}}+\widehat{\theta}_{1} \omega_{r}+\widehat{\theta}_{2}+k_{\omega} e_{\omega}\right),
$$

where $k_{\omega}$ is the positive feedback gain.

Substituting (17) into (16),

$$
\begin{aligned}
\dot{e}_{\omega} & =\frac{1}{\theta_{4}}\left(-k_{\omega} e_{\omega}-\omega_{r} \widetilde{\theta}_{1}-\widetilde{\theta}_{2}\right. \\
& +\left(\frac{3 n_{p}}{4} e_{d} e_{q}-\frac{3 n_{p}}{4} i_{d \mathrm{ref}} e_{q}\right) \tilde{\theta}_{3}-\dot{\omega}_{\mathrm{ref}} \widetilde{\theta}_{4}+\frac{3 n_{p}}{4} e_{d} \\
& \left.+\frac{3 n_{p}}{4} \widehat{\theta}_{3} i_{d \mathrm{ref}} e_{q}-\frac{3 n_{p}}{4} \widehat{\theta}_{3} e_{d} e_{q}\right) .
\end{aligned}
$$

Let

$$
\begin{aligned}
& \boldsymbol{\varphi}_{1}=\left[-\omega_{r},-1,\left(\frac{3 n_{p}}{4} e_{d} e_{q}-\frac{3 n_{p}}{4} i_{d \mathrm{ref}} e_{q}\right),-\dot{\omega}_{\mathrm{ref}}\right]^{T}, \\
& \tilde{\boldsymbol{\theta}}_{1}=\left[\widetilde{\theta}_{1}, \widetilde{\theta}_{2}, \tilde{\theta}_{3}, \widetilde{\theta}_{4}\right]^{T} .
\end{aligned}
$$

Substituting (19) into (18),

$$
\begin{aligned}
\dot{e}_{\omega} & =\frac{1}{\theta_{4}}\left(-k_{\omega} e_{\omega}+\frac{3 n_{p}}{4} e_{d}+\frac{3 n_{p}}{4} \widehat{\theta}_{3} i_{d \mathrm{ref}} e_{q}\right. \\
& \left.-\frac{3 n_{p}}{4} \widehat{\theta}_{3} e_{d} e_{q}+\widetilde{\boldsymbol{\theta}}_{1}^{T} \cdot \boldsymbol{\varphi}_{1}\right) .
\end{aligned}
$$

Step 2. By derivative of $e_{d}$ in (13), the dynamics of $d$-axis current tracking error can be obtained as

$$
\begin{aligned}
\dot{e}_{d} & =\dot{i}_{d \mathrm{ref}}-\dot{i}_{d} \\
& =\dot{i}_{d \mathrm{ref}}+\frac{R_{s}}{L_{d}} i_{d}-\frac{n_{p} L_{q}}{L_{d}} \omega_{r} i_{q}+\frac{n_{p} \psi_{f}}{L_{d}} \omega_{r}-\frac{1}{L_{d}} u_{d},
\end{aligned}
$$

where

$\dot{i}_{d \mathrm{ref}}$

$$
=\frac{4}{3 n_{p}}\left(\dot{\hat{\theta}}_{4} \dot{\omega}_{\text {ref }}+\widehat{\theta}_{4} \ddot{\omega}_{\text {ref }}+\dot{\hat{\theta}}_{1} \omega_{r}+\widehat{\theta}_{1} \dot{\omega}_{r}+\dot{\hat{\theta}}_{2}+k_{\omega} \dot{e}_{\omega}\right) .
$$

Let $R_{s}=\theta_{5}, L_{q}=\theta_{6}, \psi_{f}=\theta_{7}$, and $L_{d}=\theta_{8}$, and $\tilde{\theta}_{5}=$ $\widehat{\theta}_{5}-\theta_{5}, \widetilde{\theta}_{6}=\widehat{\theta}_{6}-\theta_{6}, \widetilde{\theta}_{7}=\widehat{\theta}_{7}-\theta_{7}$, and $\widetilde{\theta}_{8}=\widehat{\theta}_{8}-\theta_{8}$, where $\widehat{\theta}_{5}$, $\widehat{\theta}_{6}, \widehat{\theta}_{7}$, and $\widehat{\theta}_{8}$ are the estimations of $\theta_{5}, \theta_{6}, \theta_{7}$, and $\theta_{8} ; \widetilde{\theta}_{5}, \widetilde{\theta}_{6}$, $\widetilde{\theta}_{7}$, and $\widetilde{\theta}_{8}$ are the estimation errors of $\theta_{5}, \theta_{6}, \theta_{7}$, and $\theta_{8}$.

Then, (21) can be rewritten as

$$
\begin{aligned}
\dot{e}_{d}= & \dot{i}_{d \mathrm{ref}}-\dot{i}_{d} \\
= & \frac{1}{L_{d}}\left(\widehat{\theta}_{8} \dot{i}_{d \mathrm{ref}}+\widehat{\theta}_{5} i_{d}-n_{p} \widehat{\theta}_{6} \omega_{r} i_{q}+n_{p} \widehat{\theta}_{7} \omega_{r}-u_{d}\right) \\
& -\frac{1}{L_{d}}\left(i_{d} \widetilde{\theta}_{5}-n_{p} \omega_{r} i_{q} \tilde{\theta}_{6}+n_{p} \omega_{r} \widetilde{\theta}_{7}+\dot{i}_{d \mathrm{ref}} \widetilde{\theta}_{8}\right) .
\end{aligned}
$$


By derivative of $e_{q}$ in (14), the dynamics of $q$-axis current tracking error can be obtained as

$$
\dot{e}_{q}=\dot{i}_{q \mathrm{ref}}-\dot{i}_{q}=\dot{i}_{q \mathrm{ref}}+\frac{R_{s}}{L_{q}} i_{q}+\frac{n_{p} L_{d}}{L_{q}} \omega_{r} i_{d}-\frac{1}{L_{q}} u_{q},
$$

where

$$
\dot{i}_{\text {qref }}=i_{d} \dot{i}_{d}\left\{i_{d}^{2}+\left[\frac{\psi_{f}}{2\left(L_{d}-L_{q}\right)}\right]^{2}\right\}^{-1 / 2} .
$$

In terms of $R_{s}=\theta_{5}, L_{q}=\theta_{6}$, and $L_{d}=\theta_{8},(24)$ can be arranged as

$$
\begin{aligned}
\dot{e}_{q}= & \dot{i}_{q \mathrm{ref}}-\dot{i}_{q} \\
= & \frac{1}{L_{q}}\left(\widehat{\theta}_{6} \dot{i}_{q \mathrm{ref}}+\widehat{\theta}_{5} i_{q}+n_{p} \widehat{\theta}_{8} \omega_{r} i_{d}-u_{q}\right) \\
& -\frac{1}{L_{q}}\left(i_{q} \widetilde{\theta}_{5}+\dot{i}_{q \mathrm{ref}} \widetilde{\theta}_{6}+n_{p} \omega_{r} i_{d} \widetilde{\theta}_{8}\right) .
\end{aligned}
$$

For reaching the control goal and stabilizing the tracking error terms, the control inputs can be designed as follows:

$$
\begin{aligned}
u_{d}= & \widehat{\theta}_{8} \dot{i}_{d \mathrm{ref}}+\widehat{\theta}_{5} i_{d}-n_{p} \widehat{\theta}_{6} \omega_{r} i_{q}+n_{p} \widehat{\theta}_{7} \omega_{r}+k_{d} e_{d} \\
& +\frac{3 n_{p}}{4} e_{\omega}, \\
u_{q}= & \widehat{\theta}_{6} \dot{i}_{q \mathrm{ref}}+\widehat{\theta}_{5} i_{q}+n_{p} \widehat{\theta}_{8} \omega_{r} i_{d}+k_{q} e_{q}+\frac{3 n_{p}}{4} \widehat{\theta}_{3} i_{d \mathrm{ref}} e_{\omega} \\
& -\frac{3 n_{p}}{4} \widehat{\theta}_{3} e_{d} e_{\omega},
\end{aligned}
$$

where $k_{d}$ and $k_{q}$ are two positive feedback gains.

Substituting (27) and (28) into (23) and (26), respectively, we can obtain

$$
\begin{aligned}
\dot{e}_{d}= & -\frac{1}{L_{d}} k_{d} e_{d}-\frac{3 n_{p}}{4 L_{d}} e_{\omega} \\
& -\frac{1}{L_{d}}\left(i_{d} \widetilde{\theta}_{5}-n_{p} \omega_{r} i_{q} \widetilde{\theta}_{6}+n_{p} \omega_{r} \widetilde{\theta}_{7}+\dot{i}_{d \mathrm{ref}} \widetilde{\theta}_{8}\right), \\
\dot{e}_{q}= & -\frac{1}{L_{q}} k_{q} e_{q}-\frac{3 n_{p}}{4 L_{q}} \widehat{\theta}_{3} i_{d \mathrm{ref}} e_{\omega}+\frac{3 n_{p}}{4 L_{q}} \widehat{\theta}_{3} e_{d} e_{\omega} \\
& -\frac{1}{L_{q}}\left(i_{q} \widetilde{\theta}_{5}+\dot{i}_{q \mathrm{ref}} \widetilde{\theta}_{6}+n_{p} \omega_{r} i_{d} \widetilde{\theta}_{8}\right) .
\end{aligned}
$$

Step 3. The positive definite Lyapunov function candidate is defined as follows:

$$
V_{1}=\frac{1}{2} \theta_{4} e_{\omega}^{2}+\frac{1}{2} L_{d} e_{d}^{2}+\frac{1}{2} L_{q} e_{q}^{2}
$$

Derivative of the Lyapunov function yields

$$
\begin{aligned}
\dot{V}= & -k_{\omega} e_{\omega}^{2}-k_{d} e_{d}^{2}-k_{q} e_{q}^{2}+e_{\omega} \widetilde{\boldsymbol{\theta}}_{1}^{T} \cdot \boldsymbol{\varphi}_{1} \\
& -\left(i_{d} e_{d}+i_{q} e_{q}\right) \tilde{\theta}_{5}+\left(n_{p} \omega_{r} i_{q} e_{d}-\dot{i}_{q \mathrm{ref}} e_{q}\right) \tilde{\theta}_{6} \\
& -n_{p} \omega_{r} e_{d} \widetilde{\theta}_{7}-\left(\dot{i}_{d \mathrm{ref}} e_{d}+n_{p} \omega_{r} i_{d} e_{q}\right) \tilde{\theta}_{8} .
\end{aligned}
$$

Choose

$$
\begin{aligned}
\widetilde{\boldsymbol{\theta}}_{2} & =\left[\widetilde{\theta}_{5}, \widetilde{\theta}_{6}, \widetilde{\theta}_{7}, \widetilde{\theta}_{8}\right]^{T}, \\
\boldsymbol{\varphi}_{2} & =\left[-i_{d} e_{d}-i_{q} e_{q}, n_{p} \omega_{r} i_{q} e_{d}-\dot{i}_{q \mathrm{ref}} e_{q},-n_{p} \omega_{r} e_{d},\right. \\
& \left.-\dot{i}_{d \mathrm{ref}} e_{d}-n_{p} \omega_{r} i_{d} e_{q}\right]^{T} .
\end{aligned}
$$

By substituting (32) into (31), the derivative of the Lyapunov function can be simplified as follows after some mathematical computations:

$$
\dot{V}_{1}=-k_{\omega} e_{\omega}^{2}-k_{d} e_{d}^{2}-k_{q} e_{q}^{2}+e_{\omega} \widetilde{\boldsymbol{\theta}}_{1}^{T} \cdot \boldsymbol{\varphi}_{1}+\widetilde{\boldsymbol{\theta}}_{2}^{T} \cdot \boldsymbol{\varphi}_{2},
$$

where $k_{\omega}, k_{d}$, and $k_{q}$ are the positive control gains.

Suppose that

$$
\tilde{\boldsymbol{\theta}}^{T} \cdot \boldsymbol{\varphi}=\boldsymbol{e}_{\omega} \widetilde{\boldsymbol{\theta}}_{1}^{T} \cdot \boldsymbol{\varphi}_{1}+\widetilde{\boldsymbol{\theta}}_{2}^{T} \cdot \boldsymbol{\varphi}_{2}
$$

where

$$
\begin{aligned}
\widetilde{\boldsymbol{\theta}}= & {\left[\widetilde{\theta}_{1}, \widetilde{\theta}_{2}, \widetilde{\theta}_{3}, \widetilde{\theta}_{4}, \widetilde{\theta}_{5}, \widetilde{\theta}_{6}, \widetilde{\theta}_{7}, \widetilde{\theta}_{8}\right]^{T}, } \\
\boldsymbol{\varphi}= & {\left[-\omega_{r},-1,\left(\frac{3 n_{p}}{4} e_{d} e_{q}-\frac{3 n_{p}}{4} i_{d \mathrm{ref}} e_{q}\right),-\dot{\omega}_{\mathrm{ref}},\right.} \\
& -i_{d} e_{d}-i_{q} e_{q}, n_{p} \omega_{r} i_{q} e_{d}-\dot{i}_{q \mathrm{ref}} e_{q},-n_{p} \omega_{r} e_{d},-\dot{i}_{d \mathrm{ref}} e_{d} \\
& \left.-n_{p} \omega_{r} i_{d} e_{q}\right]^{T} .
\end{aligned}
$$

The final Lyapunov function is selected as

$$
V=\frac{1}{2} \theta_{4} e_{\omega}^{2}+\frac{1}{2} L_{d} e_{d}^{2}+\frac{1}{2} L_{q} e_{q}^{2}+\frac{1}{2} \tilde{\boldsymbol{\theta}}^{T} \cdot \boldsymbol{\tau}^{-1} \cdot \tilde{\boldsymbol{\theta}}
$$

where $\tau$ is an eighth-order positive definite diagonal matrix; the diagonal elements are described as $\tau_{1}, \tau_{2}, \tau_{3}, \tau_{4}, \tau_{5}, \tau_{6}, \tau_{7}$, and $\tau_{8}$.

Then, the derivative of the Lyapunov function yields

$$
\begin{aligned}
\dot{V} & =\theta_{4} e_{\omega} \dot{e}_{\omega}+L_{d} e_{d} \dot{e}_{d}+L_{q} e_{q} \dot{e}_{q}+\widetilde{\boldsymbol{\theta}}^{T} \cdot \boldsymbol{\tau}^{-1} \cdot \dot{\tilde{\boldsymbol{\theta}}} \\
& =-k_{\omega} e_{\omega}^{2}-k_{d} e_{d}^{2}-k_{q} e_{q}^{2}+\tilde{\boldsymbol{\theta}}^{T} \cdot \boldsymbol{\varphi}+\widetilde{\boldsymbol{\theta}}^{T} \cdot \boldsymbol{\tau}^{-1} \cdot \dot{\tilde{\boldsymbol{\theta}}}
\end{aligned}
$$

Letting

$$
\widetilde{\boldsymbol{\theta}}^{T} \cdot \boldsymbol{\varphi}+\widetilde{\boldsymbol{\theta}}^{T} \cdot \boldsymbol{\tau}^{-1} \cdot \dot{\tilde{\boldsymbol{\theta}}}=0
$$

we can obtain

$$
\dot{\tilde{\theta}}=-\tau \cdot \varphi .
$$


In terms of (36) and (40), the adaptive law depicting the uncertainties for the fully uncertain parameters can be written as

$$
\begin{aligned}
& \dot{\hat{\theta}}_{1}=\tau_{1} \omega_{r} e_{\omega}, \\
& \dot{\hat{\theta}}_{2}=\tau_{2} e_{\omega}, \\
& \dot{\hat{\theta}}_{3}=-\tau_{3} e_{\omega}\left(\frac{3 n_{p}}{4} e_{d} e_{q}-\frac{3 n_{p}}{4} i_{d \mathrm{ref}} e_{q}\right), \\
& \dot{\hat{\theta}}_{4}=\tau_{4} \dot{\omega}_{\mathrm{ref}}, \\
& \dot{\hat{\theta}}_{5}=\tau_{5}\left(i_{d} e_{d}+i_{q} e_{q}\right) \\
& \dot{\hat{\theta}}_{6}=-\tau_{6}\left(n_{p} \omega_{r} i_{q} e_{d}-\dot{i}_{q \mathrm{ref}} e_{q}\right), \\
& \dot{\hat{\theta}}_{7}=\tau_{7} n_{p} \omega_{r} e_{d}, \\
& \dot{\hat{\theta}}_{8}=-\tau_{8}\left(\dot{i}_{d \mathrm{ref}} e_{d}+n_{p} \omega_{r} i_{d} e_{q}\right) .
\end{aligned}
$$

By substituting (40) into (38), the derivative of the final Lyapunov function can be rewritten as

$$
\dot{V}=-k_{\omega} e_{\omega}^{2}-k_{d} e_{d}^{2}-k_{q} e_{q}^{2} \leq 0
$$

Equation (42) represents that there exists a positive definite Lyapunov function $V$ and its derivative $\dot{V}$ with respect to time being nonpositive for the closed-loop system.

Additionally, in terms of (41), the fully uncertain parameters of PMa-SynRM and load torque disturbance can be estimated and represented as

$$
\begin{aligned}
& \widehat{L}_{d}=\widehat{\theta}_{8}, \\
& \widehat{L}_{q}=\widehat{\theta}_{6}, \\
& \widehat{R}_{s}=\widehat{\theta}_{5}, \\
& \widehat{\psi}_{f}=\widehat{\theta}_{7}, \\
& \widehat{J}_{m}=\widehat{\theta}_{4}\left[\widehat{\psi}_{f}+\left(\widehat{L}_{d}-\widehat{L}_{q}\right) i_{q \mathrm{ref}}\right], \\
& \widehat{B}_{m}=\widehat{\theta}_{1}\left[\widehat{\psi}_{f}+\left(\widehat{L}_{d}-\widehat{L}_{q}\right) i_{q \mathrm{ref}}\right], \\
& \widehat{T}_{L}=\widehat{\theta}_{2}\left[\widehat{\psi}_{f}+\left(\widehat{L}_{d}-\widehat{L}_{q}\right) i_{q \mathrm{ref}}\right] .
\end{aligned}
$$

\subsection{Stability Analysis}

Theorem 1. For the dynamics of PMa-SynRM described in (5)-(7) with parameter uncertainties and external load disturbance, the proposed controller shown in (27) with the adaptive law of fully uncertain parameters given in (41) enables ensuring the tracking error signals in (12)-(14) to converge to $(0,0,0)$ asymptotically through Barbalat's Lemma.
To prove this theorem, by integration of (42) in its both sides from 0 to $+\infty$, we can give

$$
\begin{aligned}
& \int_{0}^{\infty} \dot{V}(t) d t \\
& \quad=-\int_{0}^{\infty}\left(k_{\omega} e_{\omega}^{2}(t)+k_{d} e_{d}^{2}(t)+k_{q} e_{q}^{2}(t)\right) d t .
\end{aligned}
$$

Thus,

$$
\begin{aligned}
& \int_{0}^{\infty}\left(k_{\omega} e_{\omega}^{2}(t)+k_{d} e_{d}^{2}(t)+k_{q} e_{q}^{2}(t)\right) d t \\
& \quad=V(\mathbf{0})-V(\infty) .
\end{aligned}
$$

It follows that

$$
\begin{aligned}
& \sqrt{\int_{0}^{\infty}\left(k_{\omega} e_{\omega}^{2}(t)\right) d t} \\
& \quad \leq \sqrt{\int_{0}^{\infty}\left(k_{\omega} e_{\omega}^{2}(t)+k_{d} e_{d}^{2}(t)+k_{q} e_{q}^{2}(t)\right) d t} \leq V(\mathbf{0}) \\
& \quad<\infty \\
& \sqrt{\int_{0}^{\infty}\left(k_{d} e_{d}^{2}(t)\right) d t} \\
& \quad \leq \sqrt{\int_{0}^{\infty}\left(k_{\omega} e_{\omega}^{2}(t)+k_{d} e_{d}^{2}(t)+k_{q} e_{q}^{2}(t)\right) d t} \leq V(\mathbf{0}) \\
& \quad<\infty, \\
& \sqrt{\int_{0}^{\infty}\left(k_{q} e_{q}^{2}(t)\right) d t} \\
& \quad \leq \sqrt{\int_{0}^{\infty}\left(k_{\omega} e_{\omega}^{2}(t)+k_{d} e_{d}^{2}(t)+k_{q} e_{q}^{2}(t)\right) d t} \leq V(\mathbf{0})
\end{aligned}
$$

Furthermore, $V(t)$ is uniformly continuous and $\dot{V}(t)$ is finite. Through Barbalat's Lemma [23], it gives that

$$
\lim _{t \rightarrow \infty} V(t)=0,
$$

which proves that the tracking errors $\left(e_{\omega}, e_{d}, e_{q}\right)$ can converge to $(0,0,0)$ asymptotically under full parameters' uncertainties and uncertain load torque disturbance for any initial condition. It is worth mentioning that the choices of control parameter and adaption gain are no restriction for use, except for positive definite terms. Previous studies have shown that control gains are frequently necessary to satisfy inequality relation with uncertain parameters [21] and selection of adaptation gains in multiparameter estimation situation is complicated [24] as (A3) encountered. The relaxation of control and adaptation gains is beneficial for implementation of the proposed control scheme apparently. 


\section{Proposed Luenberger Speed Observer}

Luenberger speed observer is an excellent linear estimation method. The product term of $i_{d} i_{q}$ in (4) represents the model nonlinearity of PMa-SynRM, which results in the fact that Luenberger speed observer cannot be used directly. Hence, an active flux $\psi_{a f}$ is firstly introduced to linearize (4) as follows:

$$
\psi_{a f}=\psi_{f}+\left(L_{d}-L_{q}\right) i_{q}
$$

Then, the torque equation (4) can be rewritten as

$$
T_{e}=\frac{3}{2} \frac{n_{p}}{2} \psi_{a f} i_{d}
$$

By substituting (48) into (3), we can obtain

$$
\psi_{q}=L_{q} i_{q}-\psi_{a f}+\left(L_{d}-L_{q}\right) i_{q}=L_{d} i_{q}-\psi_{a f} .
$$

In terms of (2) and (50), the components of $\psi_{\text {af }}$ in rotating $d$-q-axis coordinate system are

$$
\begin{aligned}
& \psi_{a f . d}=\psi_{d}-L_{d} i_{d}=0 \\
& \psi_{a f . q}=\psi_{q}-L_{d} i_{q}=L_{d} i_{q}-\psi_{a f}-L_{d} i_{q}=-\psi_{a f} .
\end{aligned}
$$

Equation (51) indicates that active flux is oriented on $q$-axis, which is shown in Figure 1.

Furthermore, for the mathematical model (see (5) and (6)), new input variables are introduced as

$$
\begin{aligned}
& v_{d}=\frac{n_{p} L_{q}}{L_{d}} \omega_{r} i_{q}+\frac{1}{L_{d}} u_{d}, \\
& v_{q}=-\frac{n_{p} L_{d}}{L_{q}} \omega_{r} i_{d}+\frac{1}{L_{q}} u_{q} .
\end{aligned}
$$

By substituting (52) and (51) into (5)-(7), we can get

$$
\begin{aligned}
& \frac{d i_{d}}{d t}=-\frac{R_{s}}{L_{d}} i_{d}-\frac{n_{p} \psi_{f}}{L_{d}} \omega_{r}+v_{d}, \\
& \frac{d i_{q}}{d t}=-\frac{R_{s}}{L_{q}} i_{q}+v_{q}, \\
& \frac{d \omega_{r}}{d t}=\frac{1}{J_{m}}\left(\frac{3}{2} \frac{n_{p}}{2} \psi_{a f} i_{d}-B_{m} \omega_{r}-T_{L}\right) .
\end{aligned}
$$

Equations (53)-(55) manifest that the nonlinear model of the original system has been linearized. Additionally, $\omega_{r}$ is only related to $i_{d}$ and the linearized reduced-order observer can be constructed to estimate the speed. Through utilization of the simplified rotor motion equation (55) and $d$-axis current equation (53), the reduced-order matrix equation can be given as

$$
\begin{aligned}
\left(\begin{array}{c}
\frac{d i_{d}}{d t} \\
\frac{d \omega_{r}}{d t}
\end{array}\right)= & \left(\begin{array}{cc}
-\frac{R_{s}}{L_{d}} & -\frac{n_{p} \psi_{f}}{L_{d}} \\
\frac{3 n_{p} \psi_{a f}}{4 J_{m}} & -\frac{B_{m}}{J_{m}}
\end{array}\right)\left(\begin{array}{l}
i_{d} \\
\omega_{r}
\end{array}\right)+\left(\begin{array}{l}
1 \\
0
\end{array}\right) v_{d} \\
& +\left(\begin{array}{c}
0 \\
-\frac{T_{L}}{J_{m}}
\end{array}\right) .
\end{aligned}
$$

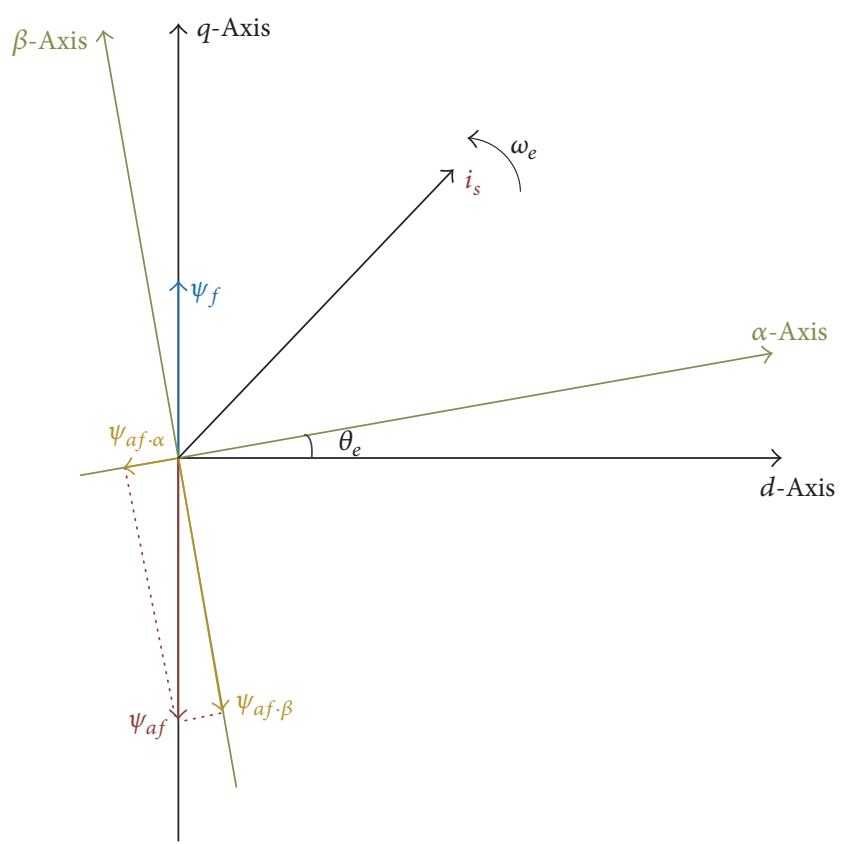

FIGURE 1: Distribution of active flux in $d q 0$ coordinated system.

For the above matrix equation, linear Luenberger observer is designed as

$$
\begin{aligned}
\left(\begin{array}{c}
\frac{d \hat{i}_{d}}{d t} \\
\frac{d \widehat{\omega}_{r}}{d t}
\end{array}\right)= & \left(\begin{array}{cc}
-\frac{R_{s}}{L_{d}} & -\frac{n_{p} \psi_{f}}{L_{d}} \\
\frac{3 n_{p} \psi_{a f}}{4 J_{m}} & -\frac{B_{m}}{J_{m}}
\end{array}\right)\left(\begin{array}{l}
\widehat{i}_{d} \\
\widehat{\omega}_{r}
\end{array}\right)+\left(\begin{array}{l}
1 \\
0
\end{array}\right) v_{d} \\
& +\left(\begin{array}{l}
\gamma \\
0
\end{array}\right)\left(i_{d}-\widehat{i}_{d}\right)+\left(\begin{array}{c}
0 \\
-\frac{T_{L}}{J_{m}}
\end{array}\right),
\end{aligned}
$$

where $\widehat{i}_{d}$ and $\widehat{\omega}_{r}$ are the estimations of $d$-axis current and speed, respectively, and $\gamma$ is the feedback gain of designed Luenberger observer.

By subtracting (57) from (56), we can get

$$
\left(\begin{array}{c}
\frac{d e_{o d}}{d t} \\
\frac{d e_{o \omega}}{d t}
\end{array}\right)=\left(\begin{array}{cc}
-\frac{R_{s}}{L_{d}}-\gamma & -\frac{n_{p} \psi_{f}}{L_{d}} \\
\frac{3 n_{p} \psi_{a f}}{4 J_{m}} & -\frac{B_{m}}{J_{m}}
\end{array}\right)\left(\begin{array}{l}
e_{o d} \\
e_{o \omega}
\end{array}\right),
$$

where $e_{o d}$ and $e_{o \omega}$ are estimation errors: $e_{o d}=i_{d}-\widehat{i}_{d}$ and $e_{o \omega}=\omega_{r}-\widehat{\omega}_{r}$.

Replacements of $B_{m} / J_{m}, \psi_{a f} / J_{m}, R_{s} / L_{d}$, and $\psi_{f} / L_{d}$ with $\widehat{\theta}_{1} / \widehat{\theta}_{4}, 1 / \widehat{\theta}_{4}, \widehat{\theta}_{5} / \widehat{\theta}_{8}$, and $\widehat{\theta}_{7} / \widehat{\theta}_{8}$, respectively, (58) can be rewritten as

$$
\left(\begin{array}{c}
\frac{d e_{o d}}{d t} \\
\frac{d e_{o \omega}}{d t}
\end{array}\right)=\left(\begin{array}{cc}
-\frac{\hat{\theta}_{5}}{\hat{\theta}_{8}}-\gamma & -n_{p} \frac{\hat{\theta}_{7}}{\hat{\theta}_{8}} \\
\frac{3 n_{p}}{4 \hat{\theta}_{4}} & -\frac{\widehat{\theta}_{1}}{\hat{\theta}_{4}}
\end{array}\right)\left(\begin{array}{l}
e_{o d} \\
e_{o \omega}
\end{array}\right) .
$$




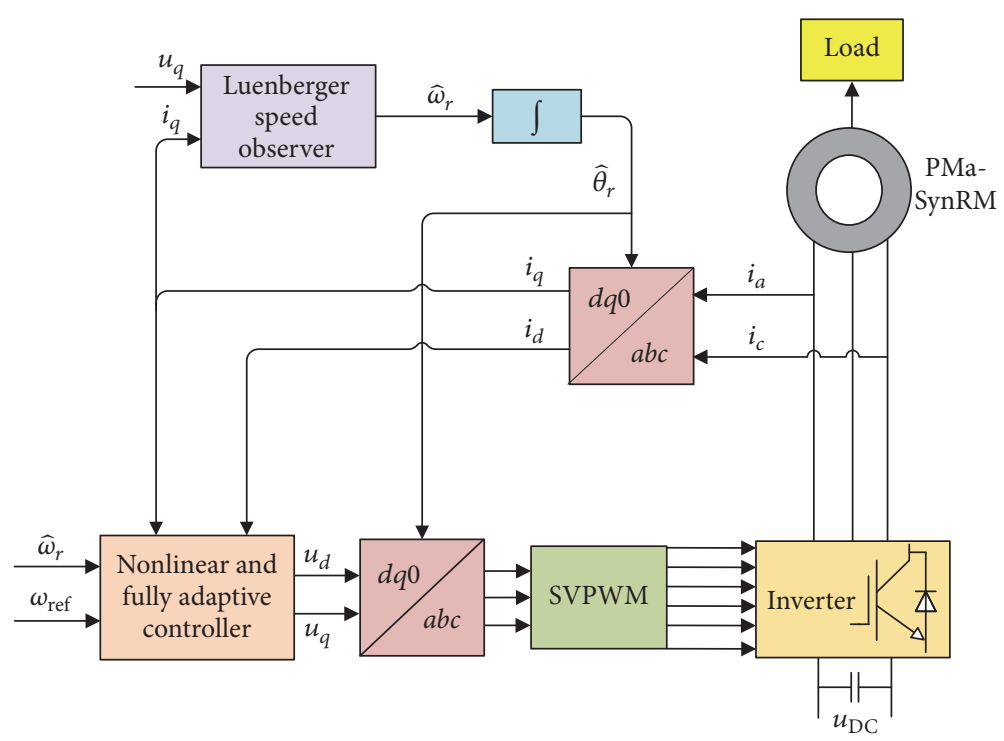

FIgURE 2: The overall configuration diagram of the proposed control scenario.

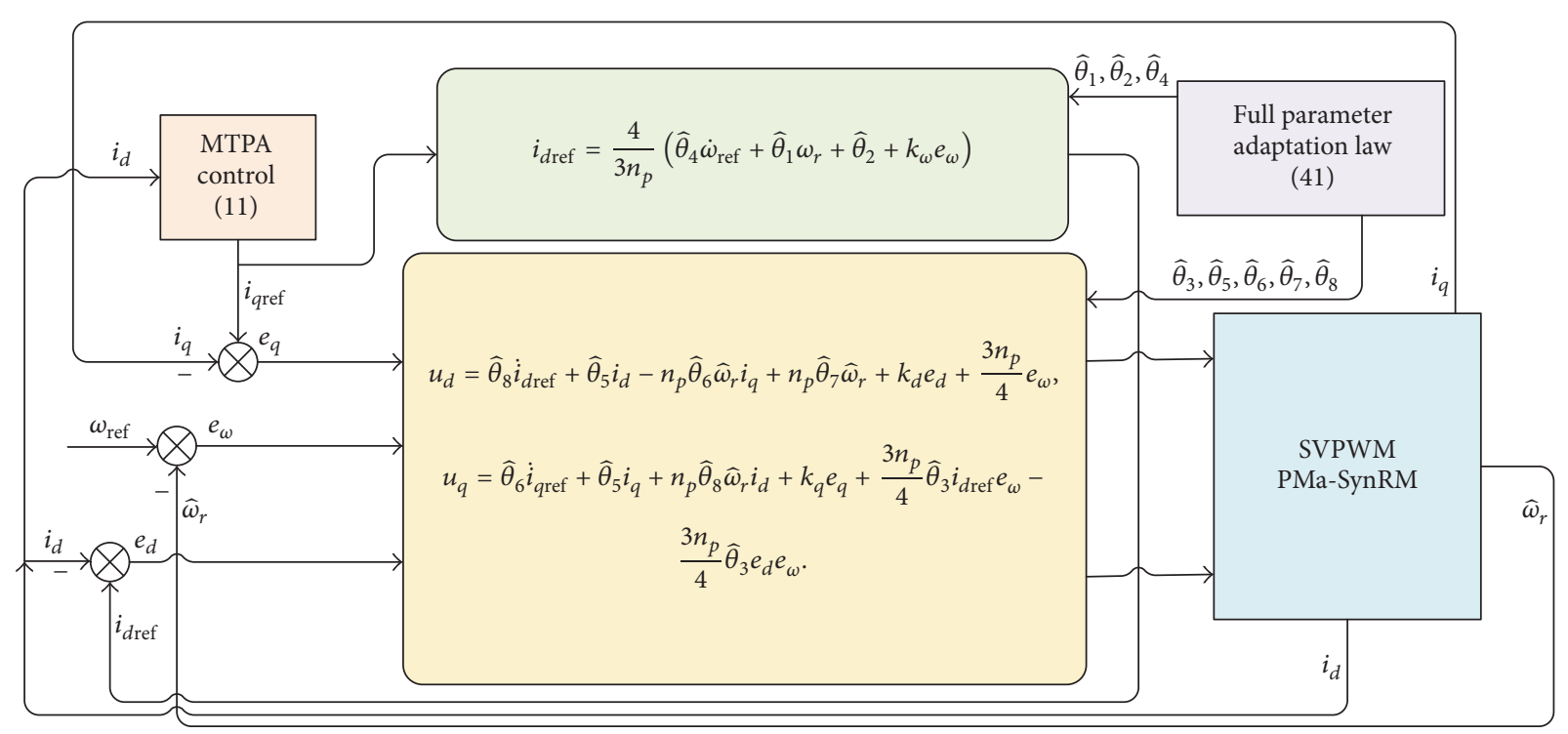

FIGURE 3: The concrete nonlinear backstepping controller with full adaptation law.

Equation (59) shows that the observed error of speed enables converging to zero through selecting a suitable parameter $\gamma$ to configurate the eigenvalue of matrix. Moreover, due to the fact that the designed speed observer is of first order, the computational burden is decreased distinctly.

\section{Digital Simulation Analysis and Discussion}

The overall configuration diagram of the proposed control scenario and concrete nonlinear backstepping controller with full adaptation law are shown in Figures 2 and 3, respectively.

The digital simulations to validate the control scheme are implemented in MATLAB and the rated and nominal parameters of PMa-SynRM are described in the following: per phase stator resistance, $2.875 \Omega, d$-axis and $q$-axis inductances, $0.133 \mathrm{H}$ and $0.058 \mathrm{H}$, respectively, viscous damping coefficient, $0.005 \mathrm{~N} / \mathrm{rad} / \mathrm{s}$, permanent magnet flux, $0.38 \mathrm{~Wb}$, moment of inertia, $0.03 \mathrm{~kg} \mathrm{~m}^{2}$, and number of pole pairs, 10 . The controller and adaption gains are selected as $k_{\omega}=75$, $k_{d}=150$, and $k_{q}=5000$, and $\tau_{1}=0.005, \tau_{2}=0.01, \tau_{3}=0.05$, $\tau_{4}=0.05, \tau_{5}=0.1, \tau_{6}=0.02, \tau_{7}=35$, and $\tau_{8}=0.01$. The feedback gain of designed Luenberger observer $\gamma$ is 20 .

To testify the superiority of the controller, adaption laws, and Luenberger speed observer adequately, the simulations consist of three groups.

Case 1. Due to the fact that very low speed regulation of PMa-SynRM is a complicated issue in control field, to validate the performance of the proposed controller and Luenberger 


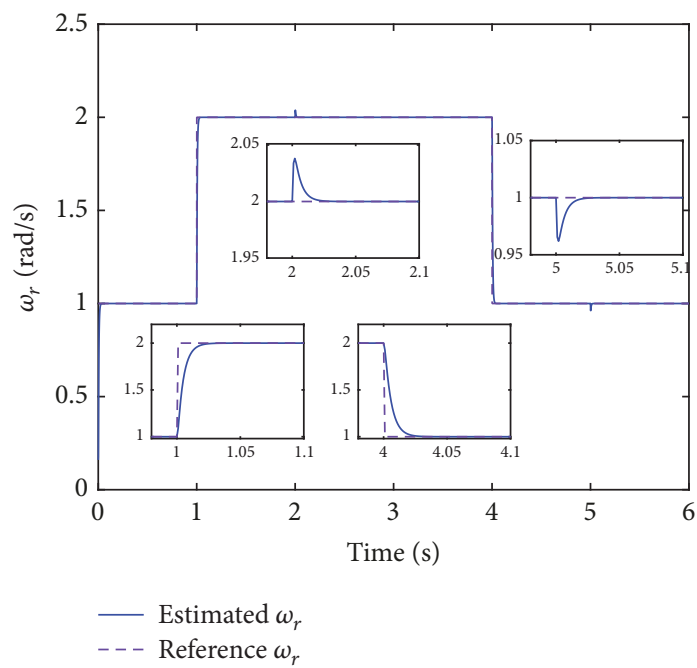

(a)

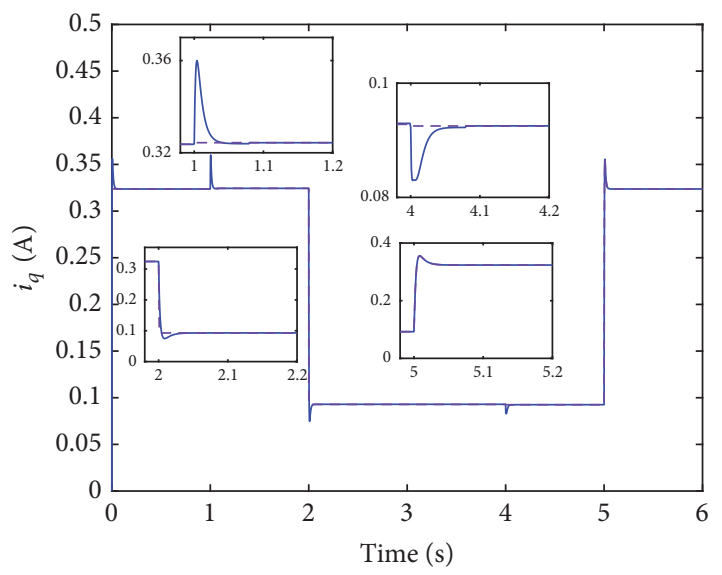

$$
-i_{q}
$$

(c)

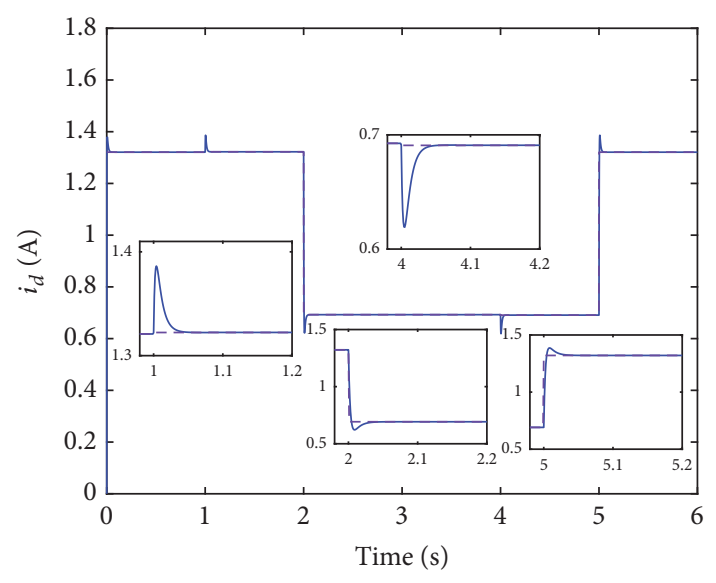

$$
-i_{d}
$$$$
\text { - - } i_{\text {dref }}
$$

(b)

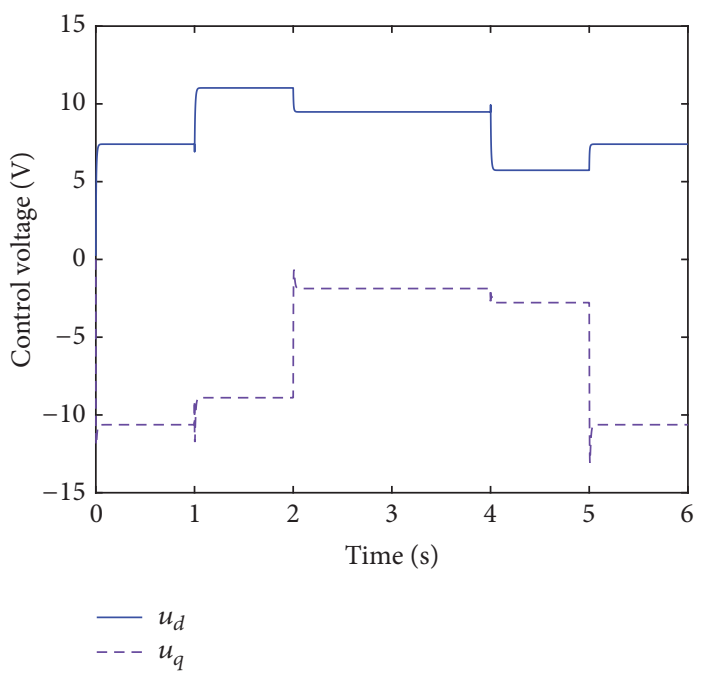

(d)

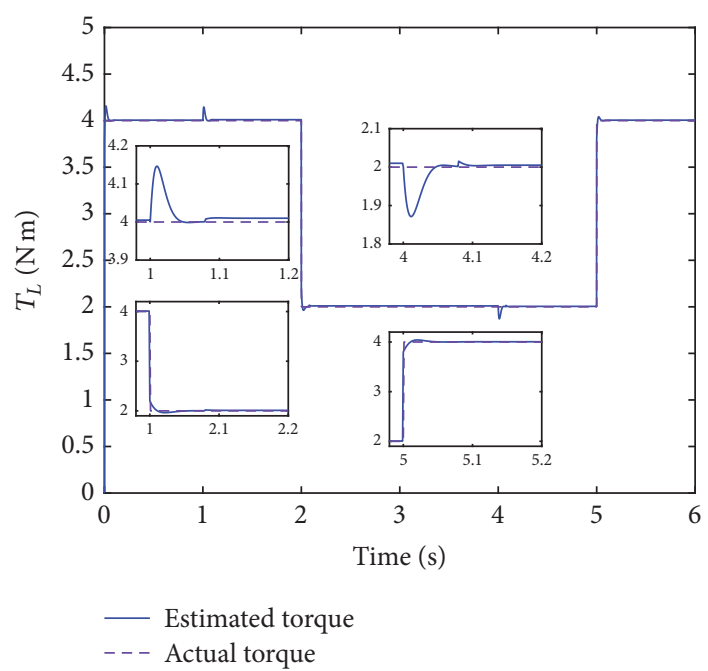

(e)

FIGURE 4: The simulation results in Case 1. (a) The estimated speed and its reference; (b) $d$-axis current and its reference; (c) $q$-axis current and its reference; (d) $d$ - $q$-axis control voltages; (e) torque and its estimation. 


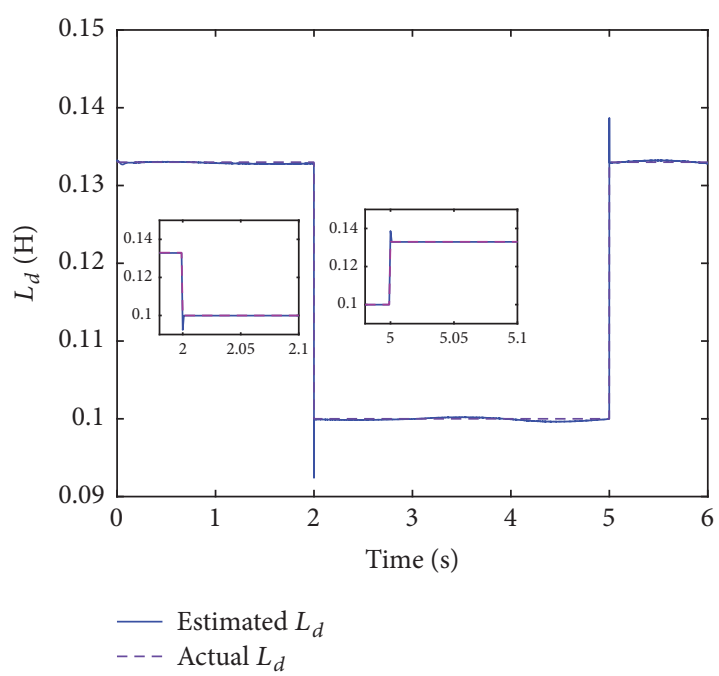

(a)

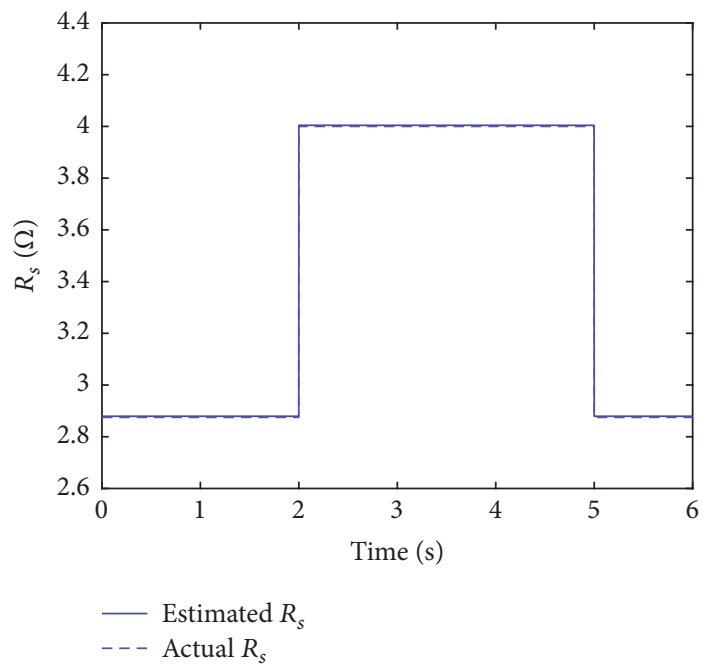

(c)

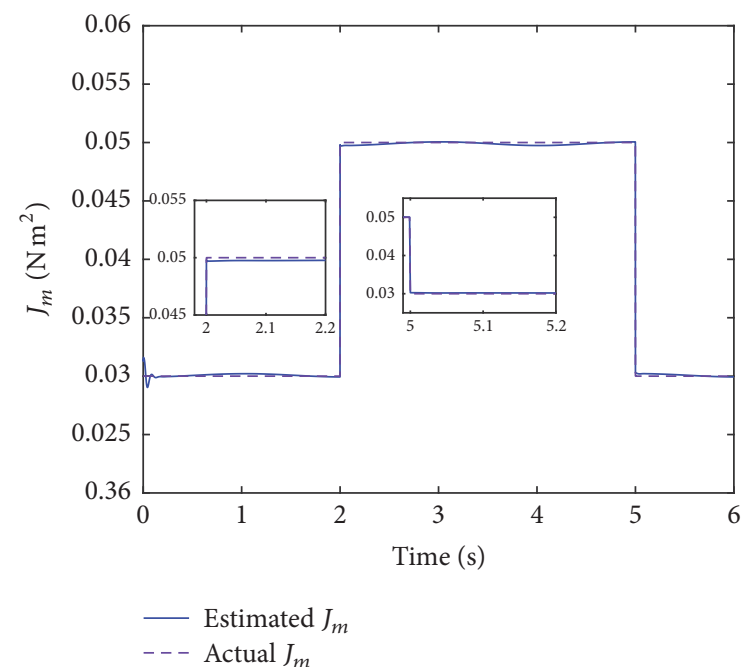

(e)

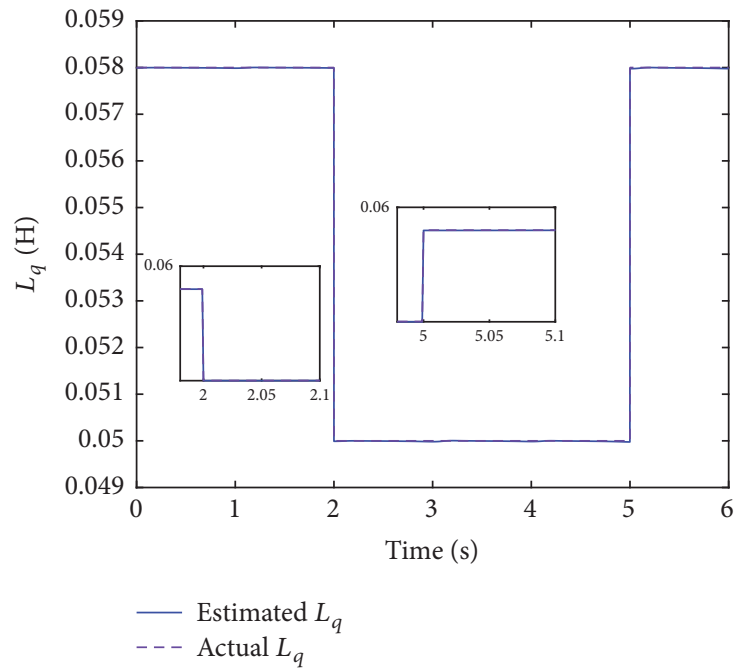

(b)

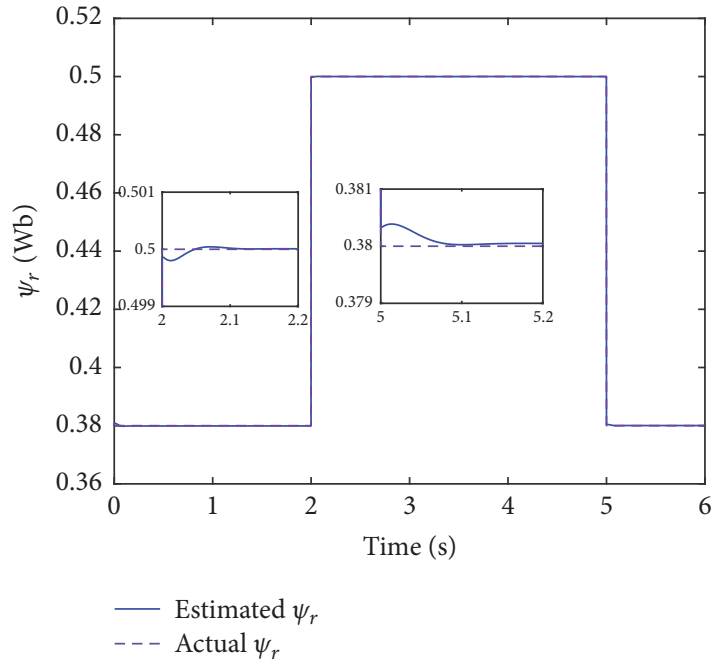

(d)

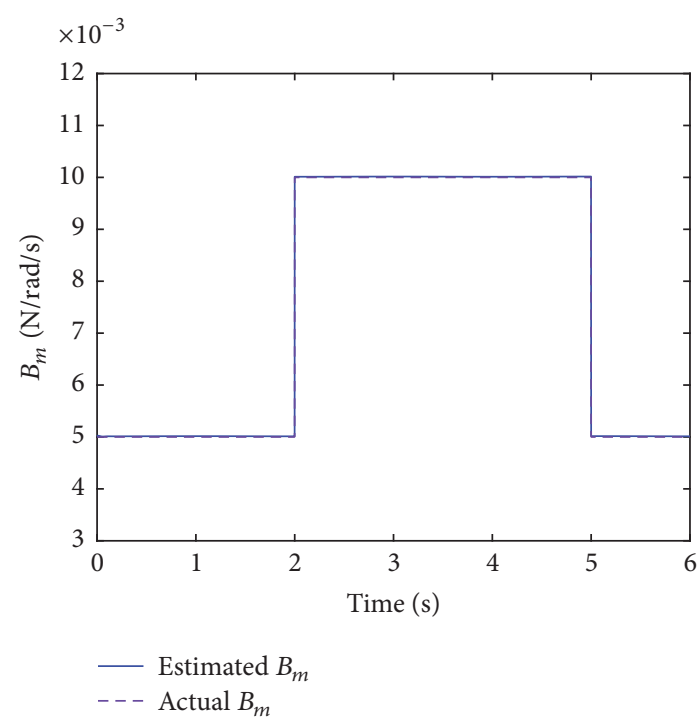

(f)

Figure 5: Continued. 


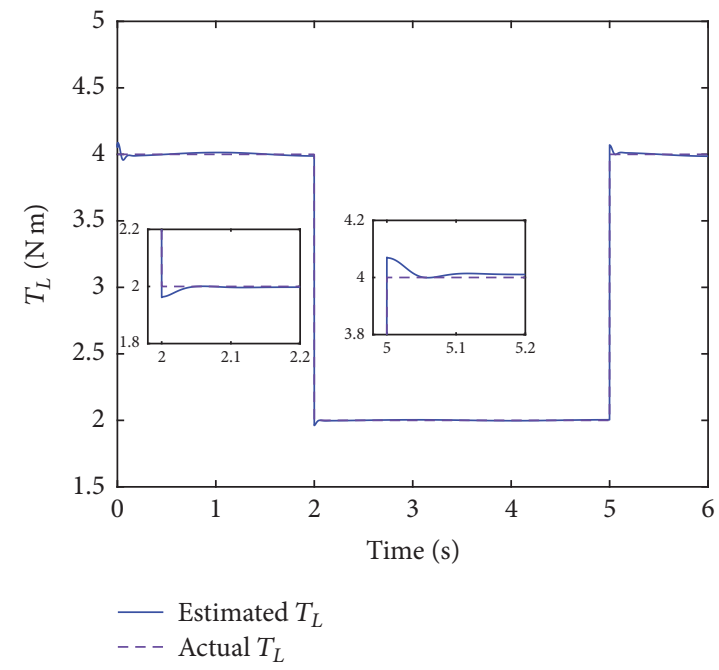

(g)

Figure 5: The simulation results in Case 2. (a) $L_{d}$ and its estimation; (b) $L_{q}$ and its estimation; (c) $R_{s}$ and its estimation; (d) $\psi_{r}$ and its estimation; (e) $J_{m}$ and its estimation; (f) $B_{m}$ and its estimation; (g) $T_{L}$ and its estimation.

speed observer, the speed reference is set to a very low and changing value, which is $1 \mathrm{rad} / \mathrm{s}$ between 0 and $1 \mathrm{~s}, 2 \mathrm{rad} / \mathrm{s}$ between $1 \mathrm{~s}$ and $4 \mathrm{~s}$, and $1 \mathrm{rad} / \mathrm{s}$ between $4 \mathrm{~s}$ and $6 \mathrm{~s}$. That is to say that the simulation time in the first simulation is $6 \mathrm{~s}$. Simultaneously, the sudden variation of load torque is taken into account and is abruptly applied to the motor as $4 \mathrm{~N} \cdot \mathrm{m}$ from 0 to $2 \mathrm{~s}, 2 \mathrm{~N} \cdot \mathrm{m}$ from 2 to $5 \mathrm{~s}$, and $4 \mathrm{~N} \cdot \mathrm{m}$ from $5 \mathrm{~s}$ to $6 \mathrm{~s}$.

The simulation results responding to the speed variation and torque disturbance are indicated in Figures 4(a)-4(e). The estimated speed and its reference are shown in Figure 4(a). The dynamic tracking responses of $d$ - $q$-axis currents and their references are in Figures 4(b) and 4(c) individually. The control inputs of $d$-q-axis voltages are displayed in Figure 4(d). The estimated load torque and its reference are demonstrated in Figure 4(e).

Figures 4(a)-4(c) indicate that the proposed controller can guarantee the output signals to track their respective references correctly and rapidly. Furthermore, Figure 4(a) verifies the effectiveness of the designed Luenberger speed observer. From Figure 4(e), the load torque is estimated precisely.

Case 2. To illustrate the capability of the adaption law and testify the robustness of the control scheme retaining the full parameter perturbations furthermore, the parameter perturbations are described as follows: per phase stator resistance increases from $2.875 \Omega$ to $4 \Omega$ at $2 \mathrm{~s}$ and changes to $2.875 \Omega$ at $5 \mathrm{~s}, d$-axis and $q$-axis inductances decrease from $0.133 \mathrm{H}$ and $0.058 \mathrm{H}$ to $0.1 \mathrm{H}$ and $0.05 \mathrm{H}$, respectively, at $2 \mathrm{~s}$ and return to their respective initial value at $5 \mathrm{~s}$, viscous damping coefficient varies from $0.005 \mathrm{~N} / \mathrm{rad} / \mathrm{s}$ to $0.01 \mathrm{~N} / \mathrm{rad} / \mathrm{s}$ at $2 \mathrm{~s}$ and returns to $0.005 \mathrm{~N} / \mathrm{rad} / \mathrm{s}$ at $5 \mathrm{~s}$, permanent magnet flux changes from $0.38 \mathrm{~Wb}$ to $0.5 \mathrm{~Wb}$ at $2 \mathrm{~s}$ and changes to $0.38 \mathrm{~Wb}$ at $5 \mathrm{~s}$, and moment of inertia increases from $0.03 \mathrm{~kg} \mathrm{~m}^{2}$ to $0.05 \mathrm{~kg} \mathrm{~m}^{2}$ at $2 \mathrm{~s}$ and changes to $0.03 \mathrm{~kg} \mathrm{~m}^{2}$ at $5 \mathrm{~s}$. Additionally, in terms of the persistency of excitation condition, a good result with parameter estimation requires abundant signals, where the speed reference is chosen as $\omega_{\text {ref }}=2 \sin (\pi t)$, and simulation time is also $6 \mathrm{~s}$. The load torque is the same as in Case 1. Figures $5(\mathrm{a})-5(\mathrm{~g})$ plot the estimated errors of fully uncertain parameters, which clarify that all the uncertain and varied parameters are estimated by the adaption law rightly. As a consequence, the simulation results shown in Figure 5 exhibit the accuracy of the adaption law and reveal the good robustness of the presented control scenario.

Simultaneously, for demonstrating the influence of each parameter variation on the presented control scheme, the dynamic responses of speed tracking are displayed in Figure 6 when changing each parameter of the following parameters: $L_{d}, L_{q}, R_{s}, \psi_{r}, J_{m}, B_{m}$, and $T_{L}$. The range of variation for each parameter is set to $\pm 50 \%$ rated value. Figure 6 indicates that the speed of the motor tracks the reference precisely with the aid of the controller and the tracking errors approach zero. Hence, the proposal controller goes against all the parameter perturbations. Merely, when the parameter deviates from its rated value, the waveform responses of speed at startup will become larger, especially for $\psi_{r}, B_{m}$, and $T_{L}$. In other words, the variations of these parameters $\psi_{r}, B_{m}$, and $T_{L}$ have a more significant influence on the speed response. However, all the speed responses in Figure 6 converge to their expected values within $0.3 \mathrm{~s}$.

Case 3. For various applications in usual, the motor operates at medium speed or high speed is indispensable. Therefore, this case indicates the simulation results in high speed condition. The speed reference is selected as $300 \mathrm{rad} / \mathrm{s}$ from zero to $1 \mathrm{~s}$ and $600 \mathrm{rad} / \mathrm{s}$ from $1 \mathrm{~s}$ to $4 \mathrm{~s}$. After $4 \mathrm{~s}$, the speed reference returns to $300 \mathrm{rad} / \mathrm{s}$. Meanwhile, the load torque changes from $4 \mathrm{~N} \cdot \mathrm{m}$ to $2 \mathrm{~N} \cdot \mathrm{m}$ at $2 \mathrm{~s}$ and returns to $4 \mathrm{~N} \cdot \mathrm{m}$ at $5 \mathrm{~s}$.

The results in this case are demonstrated in Figure 7. It is implied that, under the high speed condition, the estimated 


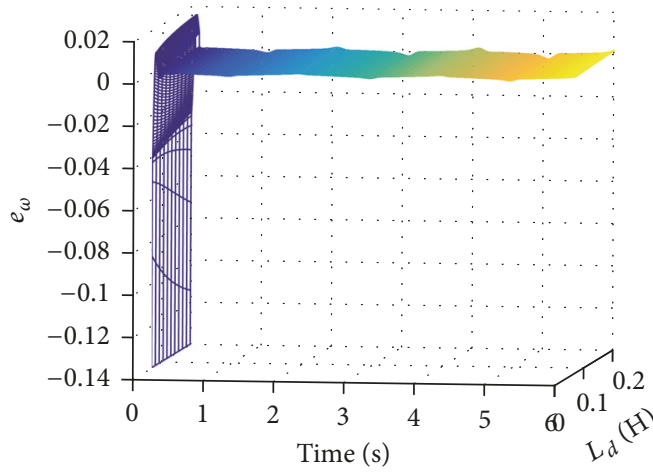

(a)

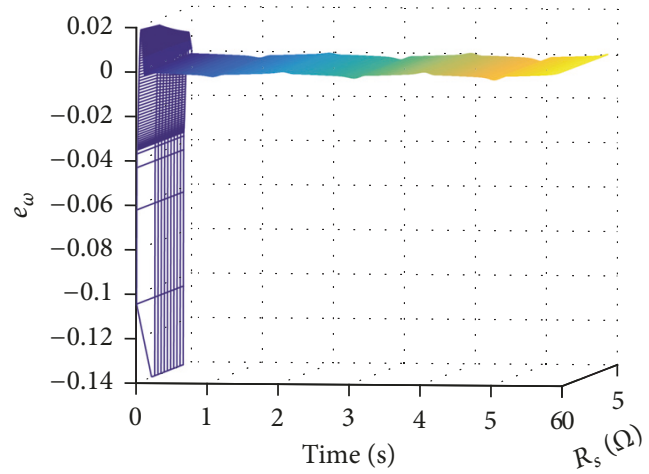

(c)

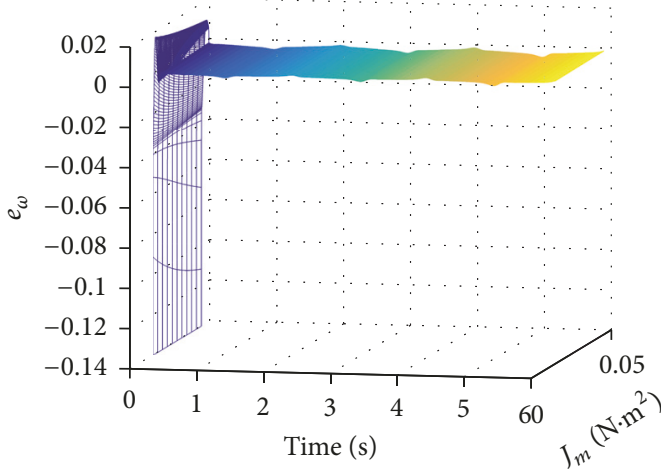

(e)

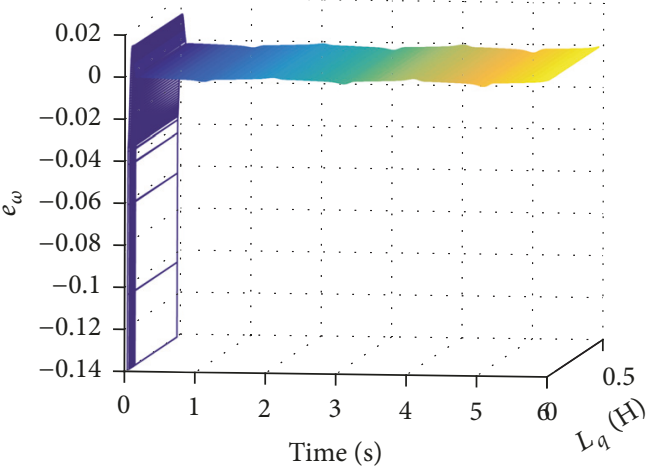

(b)

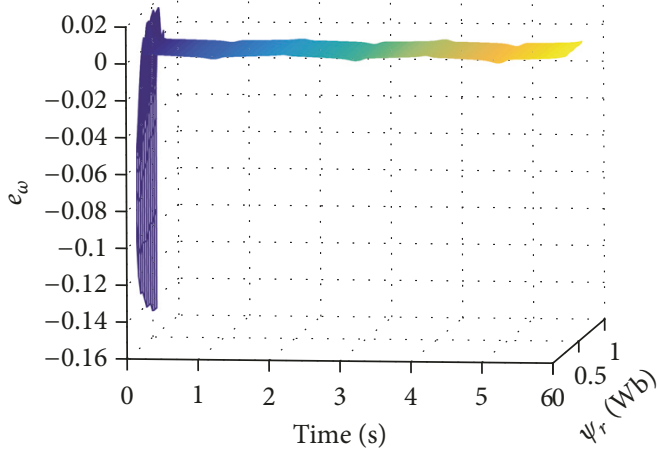

(d)

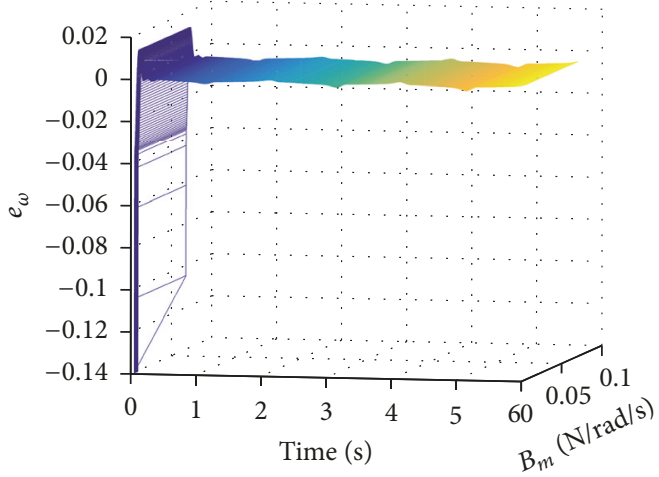

(f)

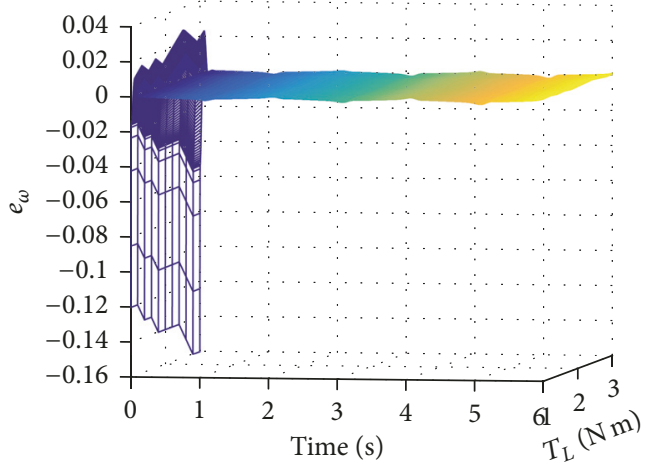

(g)

FIgURE 6: Speed tracking responses with the parameters variation. (a) Variation of $L_{d}$. (b) Variation of $L_{q}$. (c) Variation of $R_{s}$. (d) Variation of $\psi_{r}$. (e) Variation of $J_{m}$. (f) Variation of $B_{m}$. (g) Variation of $T_{L}$. 


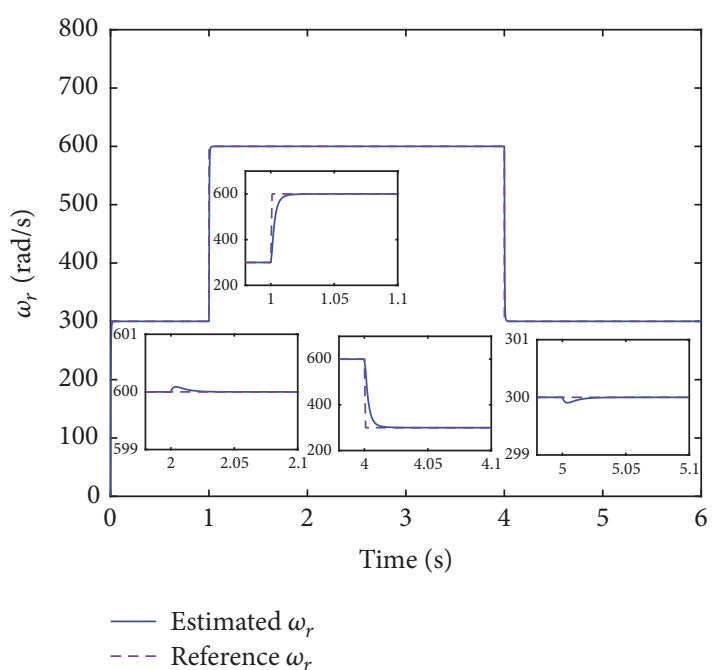

(a)

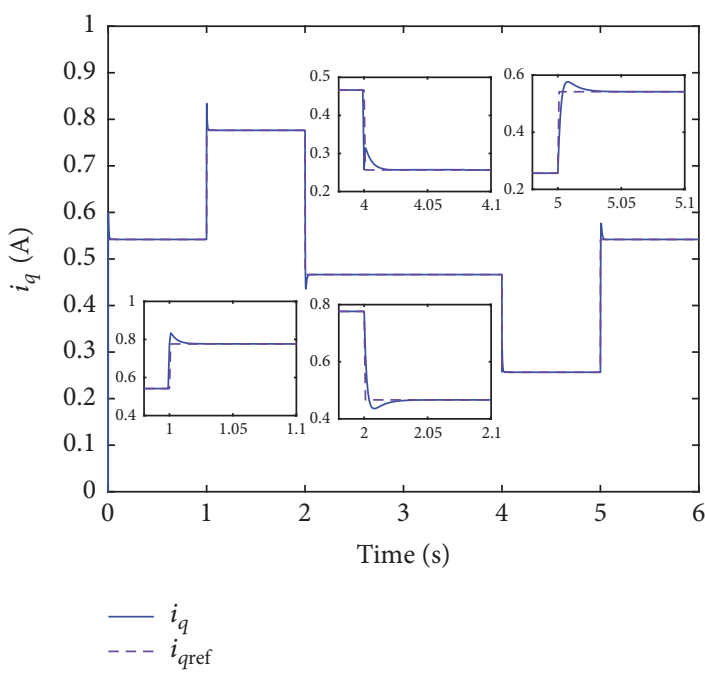

(c)

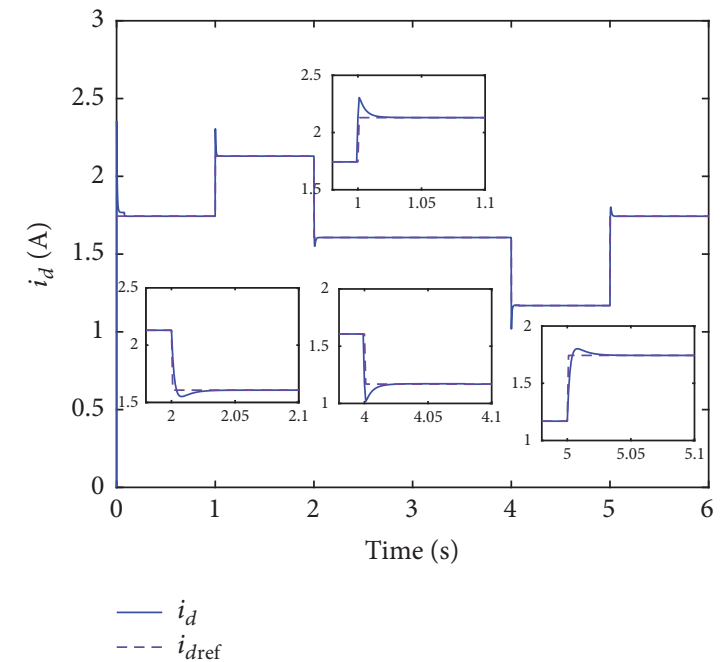

(b)

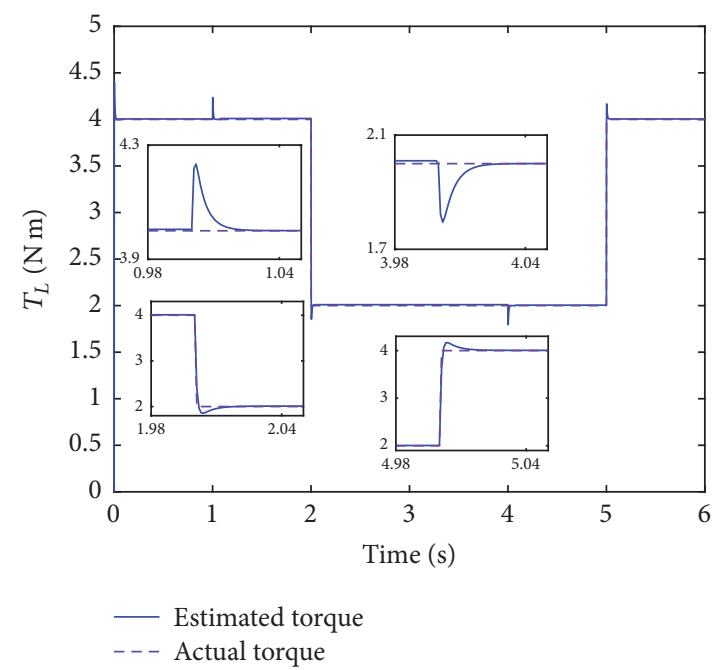

(d)

Figure 7: The simulation results in Case 3. (a) The estimated speed and its reference; (b) $d$-axis current and its reference; (c) $q$-axis current and its reference; (d) torque and its estimation.

speed shown in Figure 7(a) is also controlled to track the speed reference fast and correctly. The stator currents $i_{d}$ and $i_{q}$ are regulated to their reference signals individually.

Consequently, the presented control algorithm in the context achieves the closed-loop control of rotor speed and stator current and performs the estimations of fully unknown parameters for PMa-SynRM.

\section{Conclusions}

In this paper, on the basis of nonlinear dynamic model and parameter relation constrained in MTPA control, a nonlinear and robust adaptive backstepping speed sensorless control scheme with mismatched fully uncertain parameters is proposed for a PMa-SynRM. All the parameters of the motor and load torque are thought as uncertain except for the number of pole pairs of rotor. The design of the control scheme avoids solving complex regression matrices and the only prerequisite for all controller and adaption gains is positive definite, which reduces the workload of choice of feedback gains tremendously and ensures the asymptotic stability of the controller readily. Additionally, an active flux based Luenberger speed observer is developed. The speed observer resolves the nonlinearity of dynamic model for PMa-SynRM effectively. The proposed controller fulfills the global asymptotic tracking of a varying speed reference and $d$-q-axis current references constrained by MTPA control. Simultaneously, the designed controller is of great robustness against uncertainties in both PMa-SynRM and load torque perturbation. Digital simulations have been implemented to verify the correctness and feasibility of the proposed control scheme well. 


\section{Conflicts of Interest}

The authors declare that there are no conflicts of interest.

\section{Acknowledgments}

This work was partially supported by the National Natural Science Foundation of China (Grant no. 51407077), the Exploring Research Funds for the State Key Laboratory of Alternate Electrical Power System with Renewable Energy Sources (Grant no. LAPS2016-28), the Science and Technology Project of State Grid Corporation of China (headquarter) (Grant no. 5204BB16000F), and the Fundamental Research Funds for the Central Universities of China (Grant no. 2017MS095).

\section{References}

[1] W.-H. Kim, K.-S. Kim, S.-J. Kim et al., "Optimal PM design of PMA-SynRM for wide constant-power operation and torque ripple reduction," IEEE Transactions on Magnetics, vol. 45, no. 10, pp. 4660-4663, 2009.

[2] M. Sanada, Y. Inoue, and S. Morimoto, "Structure and characteristics of high-performance PMASynRM with ferrite magnets," Electrical Engineering in Japan (English translation of Denki Gakkai Ronbunshi), vol. 187, no. 1, pp. 42-50, 2014.

[3] E. Suárez and G. Bortolotto, "Voltage-frequency control of a self excited induction generator," IEEE Transactions on Energy Conversion, vol. 14, no. 3, pp. 394-401, 1999.

[4] I. Takahashi and T. Noguchi, "A new quick-response and high-efficiency control strategy of an induction motor ", IEEE Transactions on Industry Applications, vol. IA-22, no. 5, pp. 820827, 1986.

[5] M. Depenbrock, "Direct self-control (DSC) of inverter-fed induction machine," IEEE Transactions on Power Electronics, vol. 3, no. 4, pp. 420-429, 1988.

[6] L. Zhong, M. F. Rahman, W. Y. Hu, and K. W. Lim, "Analysis of direct torque control in permanent magnet synchronous motor drives," IEEE Transactions on Power Electronics, vol. 12, no. 3, pp. 528-536, 1997.

[7] A. Sikorski and M. Korzeniewski, "Improved algorithms of direct torque control method," Automatika - Journal for Control, Measurement, Electronics, Computing and Communications, vol. 54, no. 2, pp. 188-198, 2013.

[8] M. O. E. Aboelhassan, T. Raminosoa, A. Goodman, L. De Lillo, and C. Gerada, "Performance evaluation of a vector-control fault-tolerant flux-switching motor drive," IEEE Transactions on Industrial Electronics, vol. 60, no. 8, pp. 2997-3006, 2013.

[9] S. Kim, Y.-D. Yoon, S.-K. Sul, and K. Ide, "Maximum torque per ampere (MTPA) control of an IPM machine based on signal injection considering inductance saturation," IEEE Transactions on Power Electronics, vol. 28, no. 1, pp. 488-497, 2013.

[10] M. Preindl and S. Bolognani, "Optimal state reference computation with constrained MTPA criterion for PM motor drives," IEEE Transactions on Power Electronics, vol. 30, no. 8, pp. 45244535, 2015.

[11] C. B. Butt, M. A. Hoque, and M. A. Rahman, "Simplified fuzzy logic based MTPA speed control of IPMSM drive," IEEE Transactions on Industry Applications, vol. 40, no. 6, pp. 1529$1535,2004$.
[12] N. Yang, G. Luo, W. Liu, and K. Wang, "Interior permanent magnet synchronous motor control for electric vehicle using look-up table," in Proceedings of the 2012 IEEE 7th International Power Electronics and Motion Control Conference - ECCE Asia, IPEMC 2012, pp. 1015-1019, China, June 2012.

[13] T. Sebastian, "Temperature effects on torque production and efficiency of PM motors using NdFeB magnets," IEEE Transactions on Industry Applications, vol. 31, no. 2, pp. 353-357, 1995.

[14] P. Niazi and H. A. Toliyat, "Online parameter estimation of permanent-magnet assisted synchronous reluctance motor," IEEE Transactions on Industry Applications, vol. 43, no. 2, pp. 609-615, 2007.

[15] P. Niazi, H. A. Toliyat, and A. Goodarzi, "Robust maximum torque per ampere (MTPA) control of PM-assisted SynRM for traction applications," IEEE Transactions on Vehicular Technology, vol. 56, no. 4 I, pp. 1538-1545, 2007.

[16] M. Chen, B. Jiang, and R. Cui, "Actuator fault-tolerant control of ocean surface vessels with input saturation," International Journal of Robust and Nonlinear Control, vol. 26, no. 3, pp. 542$564,2016$.

[17] M. Wang, Z. Zhang, and Y. Liu, "Adaptive backstepping control that is equivalent to tuning functions design," International Journal of Control, Automation, and Systems, vol. 14, no. 1, pp. 90-98, 2016.

[18] G. M. Schoonhoven and M. N. Uddin, "MTPA and FW based robust nonlinear speed control of IPMSM drive using Lyapunov stability criterion," in Proceedings of the 2014 IEEE Industry Application Society Annual Meeting, IAS 2014, Canada, October 2014.

[19] Y. Jeon, S. Jung, I. Choy, and W. Cho, "Nonlinear and adaptive back-stepping speed control of IPMSM," The Transactions of the Korean Institute of Power Electronics, vol. 18, no. 1, pp. 18-25, 2013.

[20] A. K. Chakali, H. A. Toliyat, and H. Abu-Rub, "Observerbased sensorless speed control of PM-assisted SynRM for direct drive applications," in Proceedings of the 2010 IEEE International Symposium on Industrial Electronics, ISIE 2010, pp. 3095-3100, Italy, July 2010.

[21] M. Karabacak and H. I. Eskikurt, "Speed and current regulation of a permanent magnet synchronous motor via nonlinear and adaptive backstepping control," Mathematical and Computer Modelling, vol. 53, no. 9-10, pp. 2015-2030, 2011.

[22] R. Lohninger, H. Grabner, G. Weidenholzer, S. Silber, and W. Amrhein, "Modeling, simulation, and design of a permanentmagnet-assisted synchronous reluctance machine," IEEE Transactions on Industry Applications, vol. 51, no. 1, pp. 196-203, 2015.

[23] M. Krstic, I. Kanellakopoulos, and P. V. Kokotovic, Nonlinear and Adaptive Control Design, John Wiley and Sons, NY, New York, USA, 1995.

[24] J. Slotine and W. Li, Applied Nonlinear Control, Prentice-Hall, NJ, USA, 1991. 


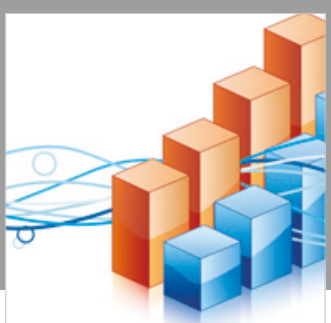

Advances in

Operations Research

\section{-n-m}
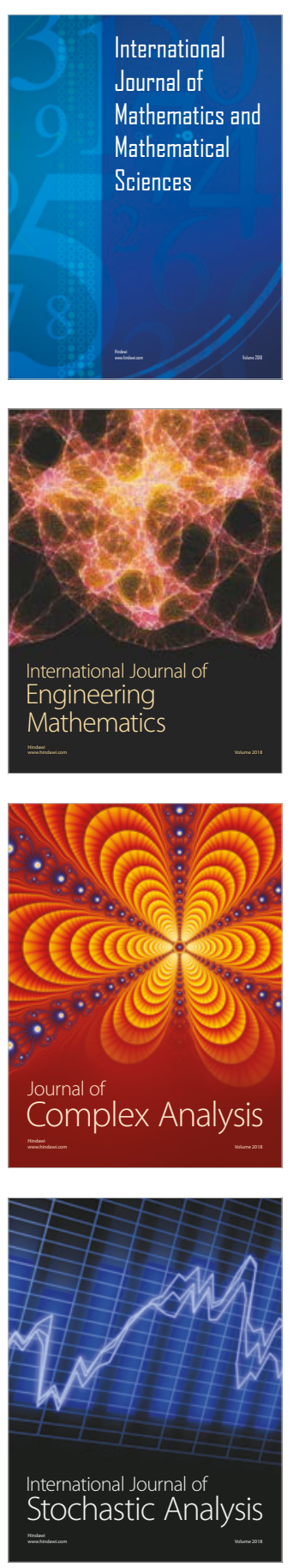
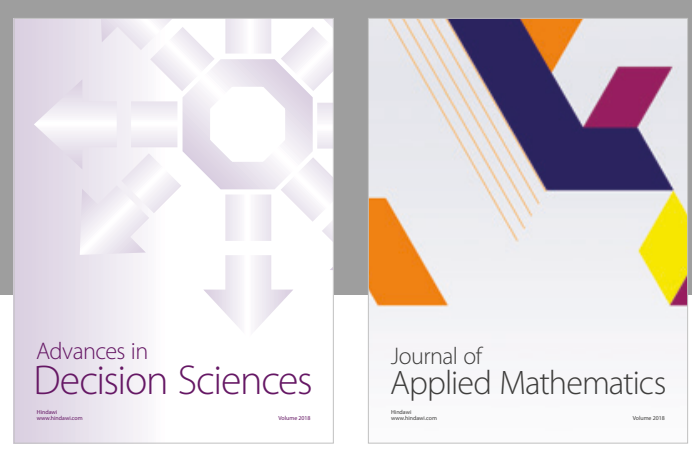

Journal of

Applied Mathematics
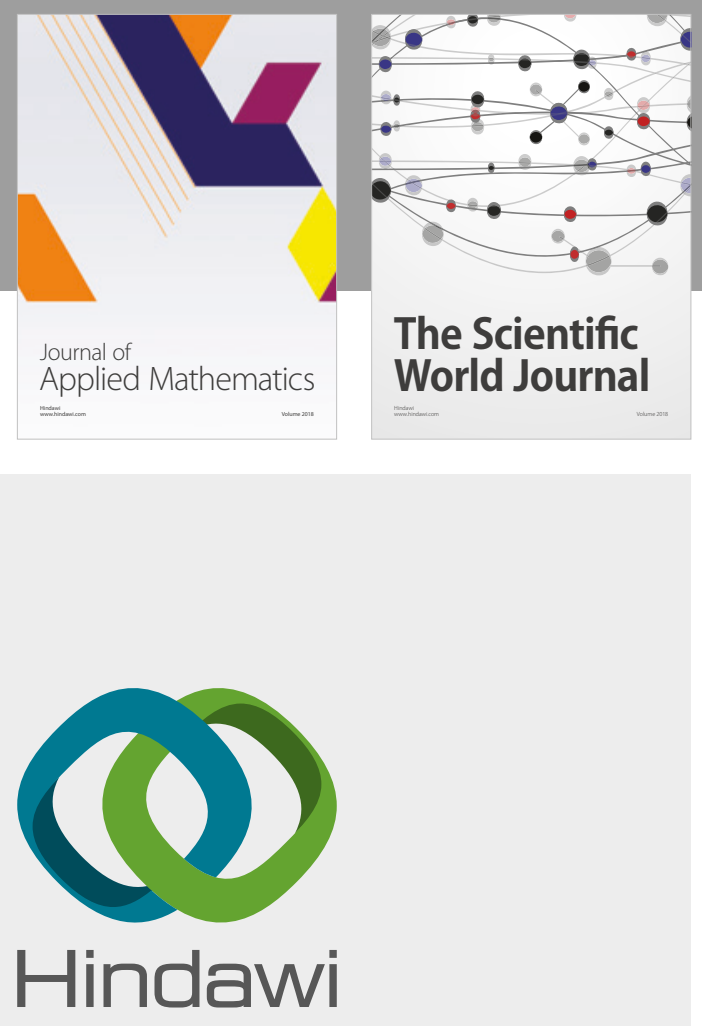

Submit your manuscripts at

www.hindawi.com

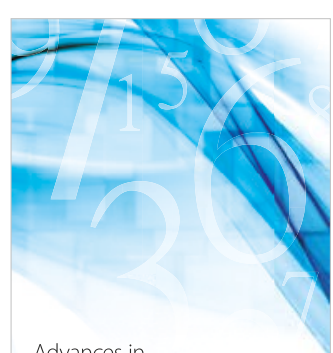

Advances in
Numerical Analysis
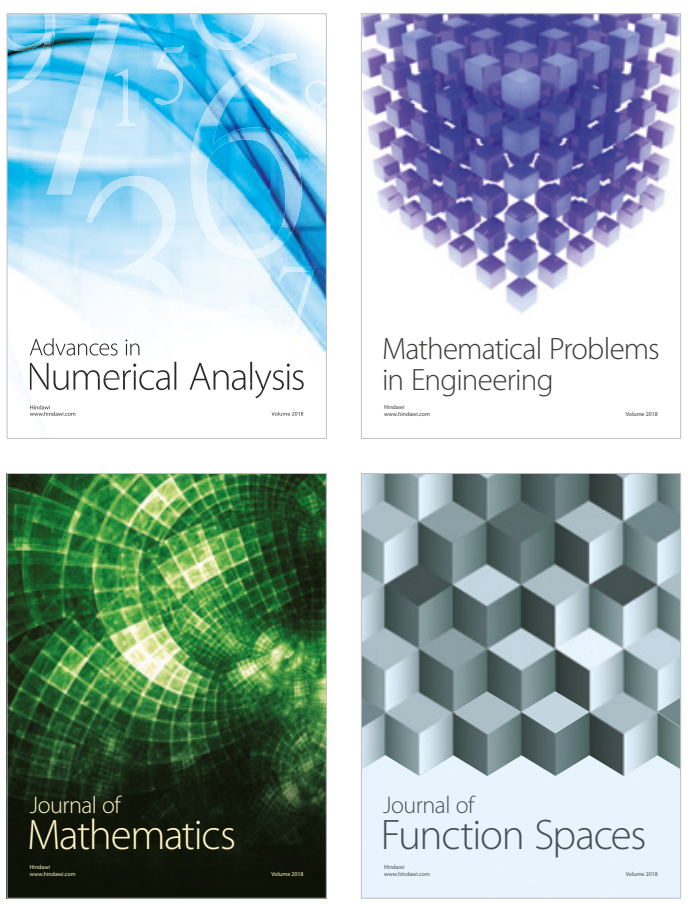

Mathematical Problems in Engineering

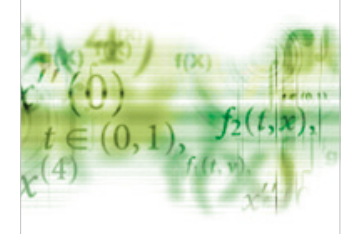

International Journal of

Differential Equations

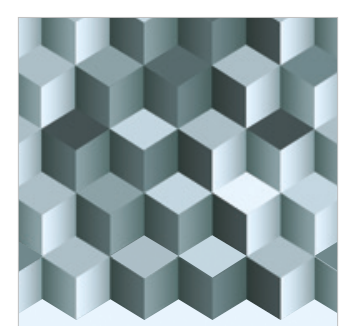

Journal of

Function Spaces

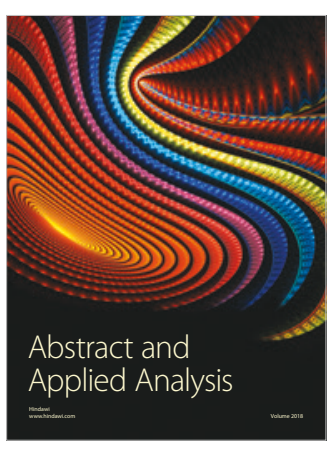

The Scientific

World Journal

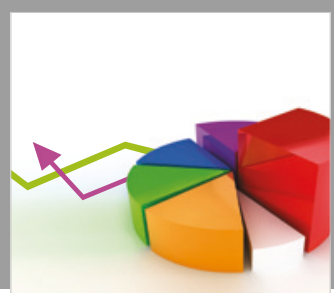

Journal of

Probability and Statistics
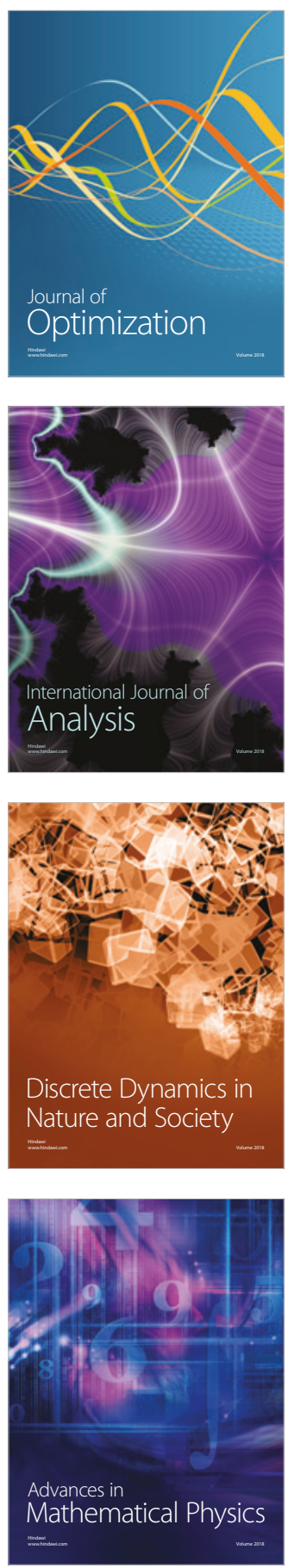